\title{
Collective Motion, Sensor Networks and Ocean Sampling
}

\author{
Naomi Ehrich Leonard, Derek Paley, Francois Lekien \\ Mechanical and Aerospace Engineering \\ Princeton University \\ Princeton, NJ 08544, USA \\ \{naomi, dpaley, lekien\}@princeton.edu \\ David M. Fratantoni \\ Woods Hole Oceanographic Institution \\ Physical Oceanography Department, MS\#21 \\ Woods Hole, MA 02543, USA \\ dfratantoni@whoi.edu
}

\begin{abstract}
This paper addresses the design of mobile sensor networks for optimal data collection. The development is strongly motivated by the application to adaptive ocean sampling for an autonomous ocean observing and prediction system. A performance metric, used to derive optimal paths for the network of mobile sensors, defines the optimal data set as one which minimizes error in a model estimate of the sampled field. Feedback control laws are presented that stably coordinate sensors on structured tracks that have been optimized over a minimal set of parameters. Optimal, closed-loop solutions are computed in a number of low-dimensional cases to illustrate the methodology. Robustness of the performance to the influence of a steady flow field on relatively slow-moving mobile sensors is also explored.
\end{abstract}

\section{INTRODUCTION}

The coupled physical and biological dynamics [1], [2] of the oceans have a major impact on the environment, from marine ecosystems to the global climate. In order to understand, model and predict these dynamics, oceanographers and ecologists seek measurements of temperature, salinity, flow and biological variables across a range of spatial and temporal scales [3], [4], [5]. Small spatial and temporal scales drive the need for a mobile sensor network rather than a static sensor array. For example, a static sensor network designed to measure an eddy that is localized and moving will necessarily be very refined and require many sensors. On the other hand, mobile sensor networks, comprised of sensorequipped autonomous vehicles, can exploit their mobility

Corresponding Author: Naomi Leonard, MAE Department, Engineering Quadrangle, Olden Street, Princeton University, Princeton, NJ 08544, Tel: +1 609258 5129, Fax: +1 6092586109 , naomi@princeton.edu

\author{
Rodolphe Sepulchre \\ Electrical Engineering \& Computer Science \\ Université de Liège \\ Institut Montefiore B28, B-4000 Liège, Belgium \\ r.sepulchredulg.ac.be \\ Russ E. Davis \\ Physical Oceanography Research Division \\ Scripps Institution of Oceanography, UCSD \\ La Jolla, CA 92093-0230, USA \\ rdavis@ucsd.edu
}

to follow features and/or monitor large areas with timevarying, spatially distributed fields, assuming that the number of vehicles and their speed and endurance are well matched to the speeds and scales of interest [6].

Our goal is to design a mobile sampling network to take measurements of scalar and vector fields ${ }^{1}$ and collect the "best" data set. A cost function, or sampling metric must be defined in order to give meaning to the term "optimal data set". For example, the performance metric that we consider in this paper defines an optimal data set as one in which uncertainty in a linear model estimate of the sampled field is minimized. A complementary approach to defining a synoptic performance metric is presented in [9]. Alternate metrics emphasize the sampling of regions of highest dynamic variability or focus on areas of high economical or strategical importance. Clearly the coordination of the sensors in the network is critical to maintain optimal data collection, independent of the metric chosen. Accordingly, coordination and collective motion play a central role in the development here. We note further that the fields to be sampled are three-dimensional, but it is reasonable to consider two-dimensional surfaces as we do in this paper. Justification for this choice is discussed further in Section IV-B.

One effective way to enable a mobile sensor network to track and sample features in a field is to use coordinated gradient climbing strategies. For instance, in ocean sampling problems, the sensor network could be used to estimate and track maximal changes in the magnitude of the gradient in order to find thermal fronts or boundaries

\footnotetext{
${ }^{1}$ The results and methods in this paper focus on a single scalar field but can be applied to multivariate fields by using appropriate weights in the cost function [7], [8].
} 
of phytoplankton patches. Such feature-tracking strategies are particularly useful for sampling at relatively small spatial scales. Boundary tracking algorithms are developed, for example, in [10], [11], [12].

On the other hand, strategies best suited for larger spatial scales are those that direct mobile sensors to provide synoptic coverage. Typically, the goal is to control the sensor network so that error in the estimate of the field of interest is minimized over the region in space and time. In this case, sensors should not cluster else they take redundant measurements. Coordinated vehicle trajectories should be designed according to the spatial and temporal variability in the field in order to keep the sensor measurements appropriately distributed in space and time.

In Section II we motivate the ocean sampling problem and state our central objective. This objective, aimed at collecting the richest possible data set with a mobile sensor network, is representative of sampling objectives in an number of domains. We describe some of the challenges that distinguish adaptive sampling networks in the ocean from networks on land, in the air or in space.

Before developing our ideas further, we next describe in Section III an ocean sampling network field experiment. The intention is both to provide inspiration for future possibilities and to illustrate a number of the practical challenges. Coordinated control strategies and gradient estimation for small-scale problems (approximately 3 kilometers) were tested on a group of autonomous underwater gliders in Monterey Bay, California in August 2003 as part of the Autonomous Ocean Sampling Network (AOSN) project [13]. The method, based on artificial potentials and virtual bodies, proved successful despite limitations in communication, control and computing and challenges associated with strong currents and great uncertainty in the relatively harsh ocean environment. We present results from this effort and discuss some of the operational constraints particular to this kind of ocean sampling network.

In a field experiment planned for August 2006 in Monterey Bay, as part of the Adaptive Sampling and Prediction (ASAP) project, a larger fleet of underwater gliders with similar operational constraints as those from 2003, will be controlled to maintain synoptic coverage of a fixed region. One primary ocean science objective is to understand the dynamics of three-dimensional cold water upwelling centers. In the remainder of this paper, we examine robust, optimal broad-scale coverage performance that we consider integral to achieving this and other science objectives. Our effort focuses on design of coordinated, mobile sensor trajectories, optimized for sampling, and stabilization of the collective to these trajectories.

In Section IV we catalog general and significant issues and challenges in sensor networks, collective motion and ocean sampling. We then summarize the issues and outline the problem addressed in this paper.

In Section V we derive and define a sampling metric based on the classical objective mapping error [14], [15], [16]. This sampling metric can be used to evaluate the sampling performance of a mobile sensor network. Likewise it can be used to derive sensor platform trajectories that optimize sampling performance. We consider coordinated patterns that are near optimal with respect to the sampling metric; that is, we select a parametrized family of solutions and define a near-optimal solution as one which optimizes the sampling metric over the parameters. In Section V we present a parametrization of solutions consisting of sensors moving in a coordinated fashion around closed curves. We parametrize the relative position of the sensors (and thus the coordinated motion of the sensors) using the relative phases of the sensors. Here the phase of a sensor refers to its angle, relative to a reference, around the closed curve on which it moves. This choice of parametrization motivates our approach to stabilization of collective motion which is tightly connected to coupled phase oscillator dynamics.

In Section VI we present models for collective motion based on a planar group of self-propelled vehicles (our mobile sensors) with steering control. We exploit phase models of coupled oscillators to stabilize and control collective motion patterns where vehicles move around circles and other closed curves, with prescribed relative spacing. We then discuss in Section VII the performance of these coordinated patterns with respect to the sampling metric. We express our sampling metric as a function of non-dimensional sampling numbers (parameters that determine the size, shape and scales in the field of interest in space and time, the speed of the vehicles and the level of measurement noise), and we determine the smallest set of parameters needed for the optimal sampling problem. We present results on optimal solutions in the case of a single vehicle moving around an elliptical trajectory in a rectangular field and in the case of two vehicles, each moving around its own ellipse. In the case of two vehicles we study the optimal sampling solution in the presence of a steady flow field with (and without) the coordinated feedback control laws of Section VI. We conclude in Section VIII and provide some discussion of ongoing and future directions. 


\section{Central Objective}

Developing models and tools to better understand ocean dynamics is central to a number of important open problems. These include predicting and possibly helping to manage marine ecosystems or the global climate and predicting and preparing for events such as red tides or El Niño. For example, phytoplankton are at the bottom of the marine food chain and are therefore major actors in marine ecosystems. They impact the global climate because they absorb enough carbon dioxide to reduce the regional temperature [17]. El Niño disrupts conditions in the ocean and atmosphere which in turn affect phytoplankton dynamics [18]. Therefore, phytoplankton can be viewed as indicators of change in the ocean and atmosphere. However, the dynamics of phytoplankton are inherently coupled to the physical ocean dynamics [19]. For example, upwelling events in the ocean bring nutrient-rich, cold water from the sea bottom to the surface where phytoplankton, which need to consume iron but also need the sun for photosynthesis, can gather and grow. Accordingly, understanding the physical oceanography and how it couples with the biological dynamics is necessary for tackling a number of important open problems [1], [2].

At present there are many effective ways to collect data on the surface of the ocean. These include, for instance, sea surface temperature measurements from satellite (or airplanes) using thermal infrared sensors, surface current measurements using high frequency radar and temperature and salinity measurements from surface drifters carrying CTD (conductivity-temperature-depth) sensors. Limited measurements under the sea surface can be made with stationary moorings or with floats that move up and down in the water column and drift with the currents. Ships that tow sensor arrays can also be used to collect data under the surface.

Autonomous underwater vehicles (AUVs), equipped with sensors for measuring the environment, are among the newest available underwater, oceanographic sampling tools [20]. With AUVs come compelling new opportunities for significantly improved ocean sensing; recent advances in technology have made it possible to imagine networks of such sensor platforms scouring the ocean depths for data [21]. Underwater gliders, described in Section III, are a class of endurance AUVs designed explicitly for collecting such data continuously over periods of weeks or even months [22], [23], [24].

What makes AUVs particularly appealing in this context is their ability to control their own motion. Using feedback control, AUVs can be made to perform as an intelligent data-gathering collective, changing their paths in response to measurements of their own state and measurements of the sampled environment. A reactive approach to data gathering such as this is often referred to as adaptive sampling. Naturally, with new resources and opportunities come new research questions. Of particular importance here is the question of how to use the mobility and adaptability of the network to greatest advantage.

Our central objective is to design and prove effective and reliable a mobile sensor network for collecting the richest data set in an uncertain environment given limited resources. This is a representative objective for mobile sensor networks and adaptive sampling problems over a number of domains. One such domain is the Earth's atmosphere where airplanes, balloons, satellites and networks of radars are used to collect data for weather observation and prediction. In space, clusters of satellites with telescopes can be used to measure characteristics of planets in distant solar systems. Sensor networks are also being developed in numerous environmental monitoring settings such as animal habitats and river systems [25]. Many of these networks use stationary sensors, although even if not mobile, the sensors can be made reactive, as in the network that was tested in Australia for soil moisture sensing and evaluation of dynamic response to rainfall events [26].

An ocean observing mobile sensor network is distinguished from many of these other applications by two significant factors. The first factor is the difficulty in communicating in the ocean. On land or in the air, it is relatively easy to communicate using radio frequency. However, radio frequency communication is not possible underwater, and it is not yet practical to use underwater acoustic communication in the settings of interest, where underwater mobile sensor platforms may be tens of kilometers apart. Communication is possible when underwater vehicles surface, which they typically do at regular intervals to get GPS updates and to relay data. However, the intervals between surfacings can be long and therefore challenging for the navigation of a single vehicle and the control of the networked system.

A second distinguishing factor is the influence of the ocean currents on the mobile sensor platforms. In the case of gliders which move at approximately constant speed relative to the flow, ocean currents can sometimes reach or even exceed the speed of the gliders. Unlike an airplane which typically has sufficient thrust to maintain course despite winds, a glider trying to move in the direction of a strong current will make no forward progress. Since the ocean currents vary in space and in time, the problem of coordinating mobile sensors becomes challenging. For instance, two sensors that should stay 
sufficiently far apart may be pushed toward each other leading to less than ideal sampling conditions.

\section{A Field EXPERIMENT In MONTEREY BAY}

The goal of the Autonomous Ocean Sampling Network (AOSN) project is to develop a sustainable, portable, adaptive ocean observing and prediction system for use in coastal environments [21]. The project uses autonomous underwater vehicles carrying sensors to measure the physics and biology in the ocean together with advanced ocean models in an effort to improve our ability to observe and predict coupled biological and physical ocean dynamics. Critical to this research are reliable, efficient and adaptive control strategies that ensure mobile sensor platforms collect data of greatest value.

\section{A. AOSN Field Experiment}

In summer 2003, a multi-disciplinary research group produced an unprecedented in situ observational capability for studying upwelling features in Monterey Bay over the course of a month-long field experiment [27]. A highlight was the simultaneous deployment of more than a dozen, sensor-equipped, autonomous underwater gliders [28], including five Spray gliders (Scripps Institution of Oceanography) and up to ten Slocum gliders (Woods Hole Oceanographic Institution), see Figure 1.

Autonomous underwater gliders are buoyancy-driven, endurance vehicles. They use pumping systems to control their net buoyancy so that they can move up and down in the ocean. Fixed wings and tail give them lift and help them to follow sawtooth trajectories in the vertical plane. Gliders can actively redistribute internal mass to control attitude. For heading control, they shift mass to roll, bank and turn (Spray) or use a rudder (Slocum). During the field experiment the gliders were configured to maintain a fixed velocity relative to the flow. Their effective forward speed was approximately $25 \mathrm{~cm} / \mathrm{s}$ (Spray) to $35 \mathrm{~cm} / \mathrm{s}$ (Slocum); this is of the same order as the stronger currents in and around Monterey Bay. Accordingly, the gliders do not make progress in certain directions when the currents are too strong.

The Spray gliders, rated to 1500 meter depth and operated to 400 meters and sometimes 750 meters during summer 2003, were deployed in deep water, traveling as far as $100 \mathrm{~km}$ offshore. The Slocum gliders, operated to 200 meter depth, were deployed closer to the coast. The gliders surfaced at regular intervals (although not synchronously) to get GPS fixes for navigation, to send data collected back to shore and to receive updated mission commands. The communication to and from

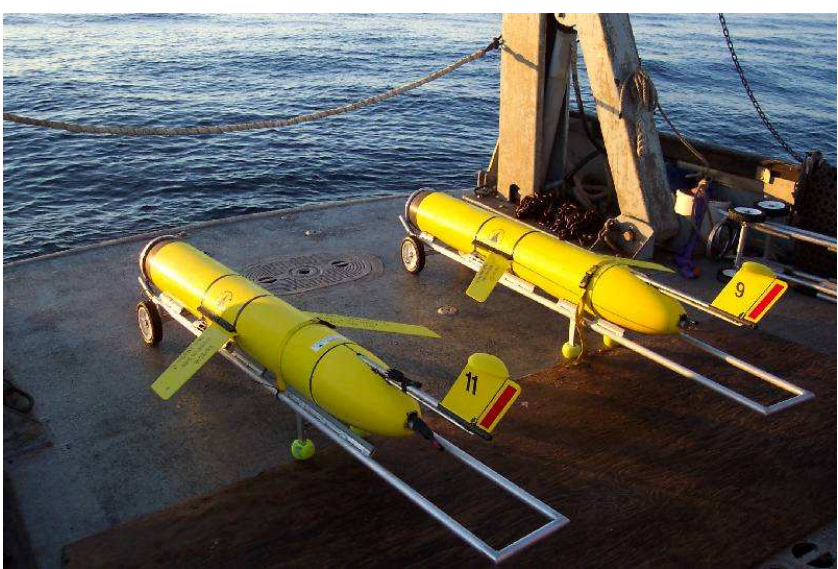

Fig. 1. Two Slocum gliders in summer 2003. Each is about 1.5 meters long. Motion in the vertical plane follows a sawtooth trajectory. A rudder is used to steer in the horizontal plane. Maximum depth is 200 meters and average forward speed relative to the flow is approximately $35 \mathrm{~cm} / \mathrm{s}$. During the AOSN 2003 experiment, the gliders were configured to surface and communicate as frequently as every two hours.

the shore computers, via Iridium satellite and ethernet, was the only opportunity for communication "between" gliders; the gliders were not equipped with means to communicate while they were underwater.

On a typical single battery cycle, the Slocum gliders performed continuously for up to two weeks between deployment and recovery while the Spray gliders remained in the water for the entire experiment (about five weeks). Collectively, the gliders delivered a remarkably plentiful data set.

Check next 2 sentences.

ToDo

Figures 2 and 3 show locations of the data collected by all of the gliders over the course of the month-long field experiment. Along its trajectory through the water, each glider records temperature, salinity, chlorophyll fluorescence (a proxy for concentration of phytoplankton) and other quantities. The set of measurements taken over one cycle of the vertical sawtooth, referred to as a profile, is assigned the single horizontal position and time corresponding to the initial, final or average position of the cycle. As the gliders move up and down, they measure temperature, salinity, chlorophyll-a fluorescence (as a proxy for phytoplankton concentration) and other physical and biological quantities depending on the installed sensors. Each point in Figures 2 and 3 represents the surface location of one profile as the glider moves along its path. Figure 2 shows the paths of the five Spray gliders traveling back and forth along lines approximately perpendicular to the shore. As seen in 


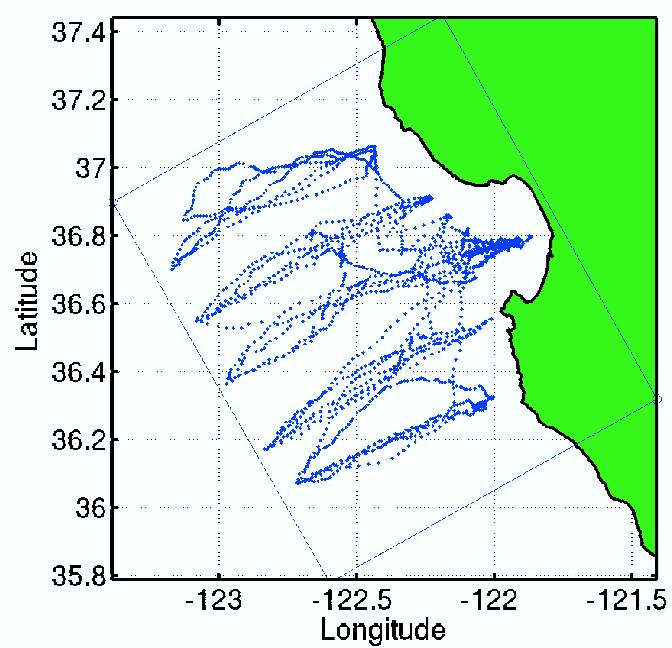

Fig. 2. Sensor measurement locations (Spray). Each point represents the location of a profile.

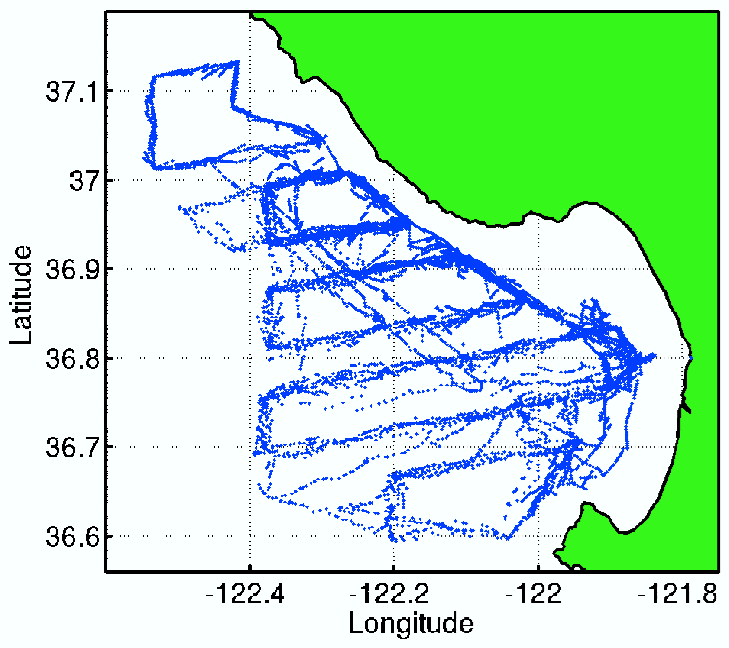

Fig. 3. Sensor measurement locations (Slocum). Each point represents the location of a profile.

Figure 3, the Slocum gliders, traveled around trapezoidal racetracks closer to shore, other than when used for coordination experiments as described next.

\section{B. Cooperative Control Sea Trials}

In this section we summarize results of sea trials, run as part of the field experiment, with small fleets of Slocum underwater gliders controlled in formations [13]. The focus was on relatively small scales in the region (on the order of 3 kilometers) and feature tracking capabilities of mobile sensor networks. The sea trials were aimed at demonstrating strategies for cooperative control and gradient estimation of scalar sampled fields using a mobile sensor network comprised of three gliders in a strong flow field with limited communication and feedback.

The control strategy was derived from the virtual body and artificial potential (VBAP) multi-vehicle control methodology presented in [29]. VBAP is a general strategy for coordinating the translation, rotation and dilation of a group of vehicles and can be used in missions such as gradient climbing in a scalar, environmental field. A virtual body is a collection of moving reference points with dynamics that are computed centrally and broadcast to vehicles in the group. Artificial potentials are used to couple the dynamics of vehicles and a virtual body so that desired formations of vehicles and a virtual body can be stabilized. Each vehicle uses a control law that derives from the gradient of the artificial potentials; therefore, each vehicle must have available the position of at least the nearest neighboring vehicles and the nearest reference points on the virtual body. If sampled measurements of a scalar field can be communicated to a central computer, the local gradients of a scalar field can be estimated. The speed of the virtual body is controlled to ensure stability and convergence of the vehicle formation. Gradient climbing algorithms can also prescribe virtual body direction. For example, the virtual body (and consequently the vehicle group) can be directed to head for the coldest water when temperature gradient estimates computed from vehicle measurements are available.

The control theory and algorithms described in [29] depend upon a number of ideal assumptions on the operation of the vehicles in the group, including continuous communication and feedback. Since this was not the case in the operational scenario of the field experiment, a number of modifications were made. Details of the modifications are described in [30]; these include accommodation of constant speed of gliders, relatively large ocean currents, waypoint tracking routines, communication only every two hours when gliders surface (asynchronously) and other latencies.

For the Slocum vehicles, each glider has on-board low-level control for heading and pitch which enables it to follow waypoints [31]. A waypoint refers to a vertical cylinder in the ocean with given radius and position. When a sequence of waypoints is prescribed, the glider follows the waypoints by passing through each of the corresponding cylinders in the prescribed sequence using its heading control. Heading control requires not only that the glider know the prescribed waypoint sequence, but also that it can measure (or estimate) its own position and heading. Heading is measured on-board the glider (as is pitch and roll). Depth and vertical speed are estimated from pressure measurements. From these measurements and some further assumptions, the glider 
estimates its linear velocity. Position is then computed by integration, using the most recent GPS fix as the initial condition. This deduced reckoning approach also makes use of an estimate of average flow, computed from the error on the surface between the glider's GPS and its dead-reckoned position.

In the cooperative control sea trials of 2003, the gliders used their low-level control to follow waypoints as per usual; however, the waypoint sequences were updated every two hours using the VBAP control strategy for coordination. VBAP was run on a simulation of the glider group using the most recent GPS fixes and average flow measurements as initial conditions. The trajectories generated by VBAP were then discretized into waypoint lists which were transmitted to the gliders when they surfaced. The approach is discussed further in [30], [13].

On August 6, 2003, a sea trial was run in which three Slocum gliders were commanded to move northwest in an equilateral triangle with inter-glider distance equal to three kilometers. The desired path of the center of mass of the vehicle group was pre-planned. The trial was run for sixteen hours, with gliders surfacing every two hours (although not at the same time). The orientation of the group was unrestricted in the first half of the sea trial and constrained in the second half of the sea trial so that one edge of the triangle would always be normal to the path of the center of mass of the group.

Snapshots of glider formations as well as glider group estimates of temperature gradient are shown in Figure 4 for the August 6, 2003 sea trial. The group stayed in formation and moved along the desired track despite relatively strong currents. Further, the gradient estimate, as seen in the figure, is remarkably smooth over time and points to the colder water, as verified from independent temperature measurements. In a second sea trial, described in detail in [13], three gliders again were controlled in an equilateral triangle formation. In this sea trial the inter-glider distance was commanded initially to be six kilometers and then reduced to three kilometers to demonstrate and test the influence of changing the resolution of the mobile sensor array. The glider network performed remarkably well despite currents with magnitude as high as $35 \mathrm{~cm} / \mathrm{s}$, which is the effective speed of the gliders.

\section{Sampling, Control and Network Issues}

The knowledge and skills accumulated during the field experiment and the success of the coordinated vehicle sea trials in 2003 provide a great deal of inspiration for further possibilities in ocean sampling networks. Indeed, another field experiment is planned for August 2006,

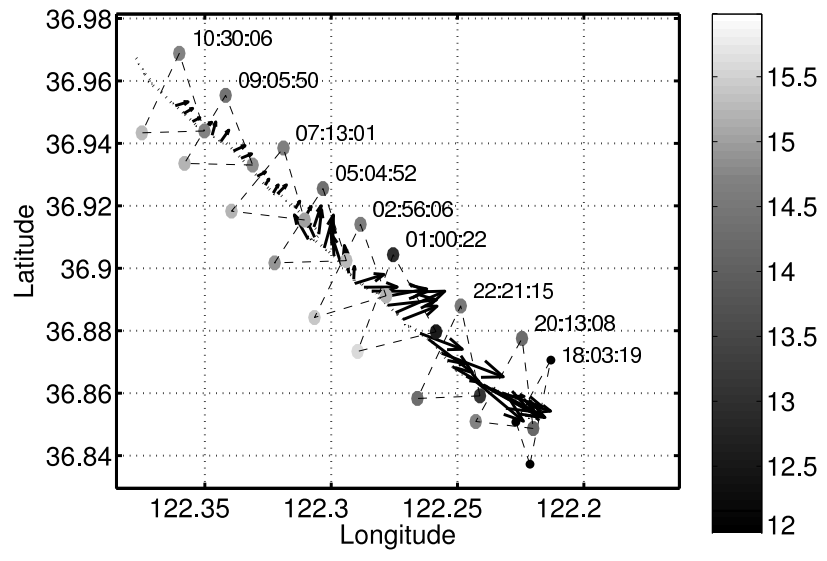

Fig. 4. Snapshots in time of glider formation starting at 18:03 UTC on August 6, 2003 and moving approximately northwest. The vectors show the estimate of minus the temperature gradient at the group's center of mass at 10 meters depth. The gray-scale map corresponds to temperature measured in degrees Celsius. The three smaller black circles correspond to the initial positions of the gliders.

again in Monterey Bay, in which a fleet of sensorequipped, autonomous underwater gliders will be operated continuously for a month as an adaptive sampling network. The fleet will include on the order of ten underwater gliders and a focus will be on broad-scale coverage of an area including the upwelling center at Point Año Nuevo (just north of Santa Cruz).

The field experiment brought experience with a number of practical challenges associated with sensor networks in the ocean, including the relatively strong flow field that pushes the vehicles around and the delays and constraints on communication.

In Section IV-A, we reflect on the broad central objective stated in Section II and list some of the important and challenging issues in sampling, control and mobile networks. In Section IV-B we clarify which issues we address in this paper and we define the boundaries of the problem addressed.

\section{A. Catalog of Challenges and Constraints}

There are a number of challenges and constraints to be investigated in order to address our central objective. The interest in optimization of data collected, management of uncertainty and extension of resources introduce conflicting demands which require trade-offs. Further, it is a goal to make the design methodology as systematic as possible since the ocean observation and prediction system should be autonomous and portable. This motivates simpler and less computationally intensive approaches. Major issues involving the performance 
metric, optimization of the metric and feedback control design for robustness include the following.

- Sampling metric definition. A metric should be selected that defines what is meant by the "best" or "richest" data set. The selected metric should be studied to evaluate how well it serves the range of goals.

- Multiple fields. When there are more than one field to be sampled, a choice needs to be made as to how to weight the importance of different fields in the sampling metric.

- Multiple scales. A complete approach to optimal ocean sampling needs to address the range of scales critical to understanding, modeling and predicting ocean dynamics. For example in the context we study, the spatial scale ranges from 25 kilometers for the synoptic picture down to 3 to 5 kilometers for features of the upwelling and even as small as hundreds of meters for some of the biology.

- 2D versus 3D. In the event that sampling in threedimensional space is desired, any methodologies derived for two dimensions need to be extended.

- Sampling metric computation and adaptation. A methodology should be developed for computing the metric with minimal computational burden and for computing inputs to the metric that are not directly measured and/or that change over time. The trade-off between optimization of the metric versus computation of the metric may need to be considered in the design and real-time control of optimal collective motion.

- Optimal, collective motion. An approach to optimizing the sampling metric should be developed so that optimal, collective motion for the mobile sensor network can be designed. Low frequency feedback measurements can be used to adapt the optimal collective motion to the changing fields, changing ocean processes, changing operational conditions and health of the sensors in the network.

- Flow field. Whether or not its components are scalar fields of specific interest, the flow field directly influences sampling performance because it can push the sensors around and prevent them from carrying out optimal sampling strategies. Accordingly, the flow field must be considered in the design of optimal, collective motion. A methodology to exploit available estimates or predictions of the flow field is of significant interest.

- Feedback control of collective motion. Relatively high rate feedback control strategies that stabilize optimal collective motion are necessary to ensure robustness of optimal sampling strategies not only with respect to the external flow field but also to other disturbances and uncertainties in the ocean environment.

Additionally, there are a number of issues associated with the sensor platforms themselves and their network operation. A list of these such issues follows.

- Constant speed. Strategies for collective motion must take into account that gliders effectively operate at constant speed (relative to the flow field). Otherwise, patterns may be designed that are not realizable. Gliders can also be operated as virtual moorings which may be applicable to the adaptive sampling problem but is not considered here.

- Transit and irregular events. There will be a significant period of time when mobile sensors are "in transit," meaning that they are on their way between optimal strategies. For example, when gliders are first deployed they should transit to locations where they will initiate their optimal strategy. However, gliders are slow and the period of time it will take to get to these locations may be significant. Therefore, their paths should be designed both to optimize sampling during transit and to minimize transit time. Similar strategies should be developed in case a mobile sensor encounters a region it must avoid (e.g. due to fishing), is taken out of the water for whatever reason, experiences a debilitating failure, etc.

- Heterogeneous groups. In case mobile sensors in the network differ in speed, endurance, sensors, etc., methodologies should be developed to exploit the differing strengths and potential roles of the sensors in the network. For instance, slow, endurance vehicles might be more useful for larger scales whereas faster, shorter-lived vehicles might serve better collecting data over smaller scales.

- Extending lifetime of sensors. Underwater gliders are designed to be endurance vehicles, a central objective being to collect data continuously over weeks or even months at a time. Accordingly, keeping energy use to a minimum is critical. This implies also keeping volume (and therefore mass) to a minimum. There is a direct trade-off here with improving sensing, navigation, communication and control. For example, communication on the ocean surface makes possible coordinated control of the sensors. However, surfacings that are too frequent can be costly in terms of energy expenditure and loss of time collecting data, whereas surfacings that are too infrequent yield very long feedback sam- 
pling periods which can diminish the performance and robustness of the control.

- Communication. Communication between gliders is done above the surface on a central computer. Coordinated control strategies for the network of sensors that are originally designed assuming continuous control will need to be revisited. Since minimizing the frequency of surfacings is desirable to minimize energy and maximize time spent collecting undersea data and minimize exposure, it is of interest to determine the maximum tolerable feedback sampling period that does not degrade overall sampling performance.

- Asynchronicity. Strategies will need to accommodate asynchronicity in time of surfacing and communication. Because the gliders will not surface at the same time, information communicated to a glider about any of the other gliders will necessarily be old.

- Latencies. It may not always be possible to close the feedback loop on the surface. For example, in the sea trials of 2003, described in Section III-B, data retrieved from a glider at its surfacing could not be used in the mission update to the glider at that same surfacing. Instead the data was used to compute new instructions communicated to the glider at the next surfacing. This introduces significant delays that need to be accommodated.

- Computing. While low-level control is computed on board the gliders, coordinated control of the network is computed on the central shore computer where inter-glider communication occurs. Possibilities for further exploiting on-board computation and local measurements should be investigated.

\section{B. Problem Definition}

In this paper we address sampling a single time- and space-varying scalar field like temperature using mobile platforms like gliders. Emphasis is on how to operate such vehicles, either singly or in coordinated fleets, to provide the most information about this field. Since the main operational control is over course and speed, we focus on mapping a single 2D horizontal field. Ultimately the data would be used to describe the 3D field either directly by analysis of 3D data or by assimilating data into high-resolution dynamical ocean models [32], [33], [34]. However, because ocean scales are similar through the upper ocean column and horizontal position is the main control variable, the $2 \mathrm{D}$ problem suffices.

To measure how well a given sampling array describes the variable of interest, we adopt a simple metric based on objective analysis (linear statistical estimation based on specified field statistics). This metric (defined in Section V-A) specifies the statistical uncertainty of the model as a function of where and when the data is taken. Since reduced uncertainty implies better measurement coverage, we also refer to this as a coverage metric. In ongoing work [2], [35], [36], [37], information that can be inferred from a dynamical model is included into the metric used to control vehicles.

We frame the optimal collective motion problem and define our approach to design of a (near) optimal mobile sensor network in Section V. By near optimal solutions, we mean that we optimize over a parametrized family of structured solutions. For example, we consider a family of closed curves parametrized by number, location, dimension and shape as well as the relative phases of the vehicles moving around these curves. This parametrization is discussed in Section V-D. The relative phases provide a low-dimensional parametrization of relative position of the vehicles and they make a connection between the optimized trajectories and the coupled phase oscillator models that we use in our coordinated control law.

We pay particular attention to gliders moving around ellipses for several reasons. First, the various periodic trajectories appropriate for oceanographic sampling (e.g., moving back and forth on a line or around a trapezoid as shown in Figures 2 and 3 from the 2003 AOSN field experiment) can be reasonably approximated with ellipses by tuning the eccentricity. Second, ellipses are minimally parameterized, smooth shapes for which we have developed a control theoretic framework. In ongoing work not presented here, we have generalized our control framework to a class of curves known as superellipses, which includes circles, ellipses and rounded rectangles. By considering superellipses and optimizing over the parameters that define them, we aim to go beyond the hand-crafted trajectories of previous experience, to automate the design, adaptation and control of sensor patterns that yield maximally information rich data sets.

In the case of gliders moving with constant speed around circles, the difference in heading for any pair of gliders can be interpreted as the relative phase of that pair of gliders. For example, if for a pair of gliders moving around the same circle, the difference in heading is 180 degrees, then the relative phase is 180 degrees and the gliders are always at antipodal points on the circle. For ellipses, the relative phase is not necessarily equivalent to the relative heading.

In Section VI we present feedback control laws that stabilize these kinds of collective motions for gliders moving at constant (unit) speed on the plane. We focus 
on the case that there may be multiple ellipses and multiple vehicles per ellipse. The objective is to ensure that gliders move around their (optimally located, oriented, sized) ellipses with optimal relative phases. In Section VII we compute and study optimal solutions and we discuss robustness of the solutions with respect to the coverage metric. We also investigate the influence of the flow field on the design and control of optimal sampling trajectories.

In this paper we assume a homogeneous group of mobile sensors. We do not address the issue of transit and irregular events; preliminary results on minimal time and minimal energy glider paths computed using forecasts of ocean flow fields are presented in [38]. We also do not address the problems in communication, asynchronicity, latency and computing described above. In [30], [13] it is discussed how these issues were handled in AOSN 2003. In [39] a control law is presented that explores extended sensing, computing and control on-board a glider. In this paper we let each sensor compute its own control law locally and we assume continuous feedback control with continuous communication without delay or asynchronicity. Because communication is not limited to neighboring gliders in the operational scenario, we assume an all-to-all interconnection topology.

A number of the issues listed in Section IV-A remain important open problems and a number are the subject of ongoing work.

\section{SAMPLing Metric And Optimality}

\section{A. Sampling Metric}

In this section, we derive a metric to quantify how well an array of gliders samples a given region. Recall that an objective is to assimilate the data in an ocean model. Therefore, the metric should reflect how a particular collected data set reduces the error in the model. This notion is necessarily dependent on the specific model or assimilation scheme used. During AOSN 2003, the data was assimilated in several high resolution ocean models [32], [33], [34] and the performance of the sampling array was similar for each. Since reliable nowcasts and forecasts of the ocean require concurrent ocean models mutually validating their results and the data requirements of these models are similar, it is natural to derive the performance metric on a simpler, more general assimilation scheme. This approach also has the advantage of avoiding the complexity and computational effort required to study specific high resolution models [40], [41]. To compute our metric we need only a background covariance function to describe the field and the locations and times corresponding to where and when the data was collected; the measurements and forecast of the field are not needed.

We consider a simple data assimilation scheme called Objective Analysis ${ }^{2}$ [42], [43]. In this framework, the scalar field (e.g., temperature, salinity) observed at each point $\mathbf{r}$ and at each time $t$ is viewed as a random variable $T(\mathbf{r}, t)$ or an ensemble of possible realizations. The algorithm provides an estimate for the average and the error variance of this estimate assuming we have an a priori description of the field, usually the mean $\bar{T}$ and the covariance $B$ of fluctuations around the mean:

$$
\begin{aligned}
& \bar{T}(\mathbf{r}, t)=\mathbf{E}[T(\mathbf{r}, t)], \\
& B\left(\mathbf{r}, t, \mathbf{r}^{\prime}, t^{\prime}\right)=\mathbf{E}\left[[T(\mathbf{r}, t)-\bar{T}(\mathbf{r}, t)]\left[T\left(\mathbf{r}^{\prime}, t^{\prime}\right)-\bar{T}\left(\mathbf{r}^{\prime}, t^{\prime}\right)\right]\right]
\end{aligned}
$$

where $\mathbf{E}[\cdot]$ represents the expected value of a random variable. The "diagonal" elements $B(\mathbf{r}, t, \mathbf{r}, t)$ contain the variance of $T(\mathbf{r}, t)$ around its expected value $\bar{T}(\mathbf{r}, t)$. We note that the assumed value of $\bar{T}$ is needed for the estimate of the field but not for the error variance of this estimate and therefore not for the performance metric that we will define.

The data collected by the gliders is a sequence of $M$ measurements $T_{k}$ at discrete points $\left(\mathbf{r}_{k}, t_{k}\right)$. Objective analysis provides an estimate $\hat{T}(\mathbf{r}, t)$ of the field $T(\mathbf{r}, t)$ as a linear combination of all the data:

$$
\hat{T}(\mathbf{r}, t)=\bar{T}(\mathbf{r}, t)+\sum_{k=1}^{M} \zeta_{k}(\mathbf{r}, t)\left[T_{k}-\bar{T}\left(\mathbf{r}_{k}, t_{k}\right)\right],
$$

where the $M$ coefficients $\zeta_{k}$ minimize the least square uncertainty of $\hat{T}(\mathbf{r}, t)$. While the problem for each pair $(\mathbf{r}, t)$ is separate from others, the coefficients $\zeta_{k}(\mathbf{r}, t)$ minimize the mean square error integrated over the region and period of interest:

$$
\int d \mathbf{r} \int d t \mathbf{E}[[T(\mathbf{r}, t)-\hat{T}(\mathbf{r}, t)][T(\mathbf{r}, t)-\hat{T}(\mathbf{r}, t)]] .
$$

The optimal coefficients are

$$
\zeta_{k}(\mathbf{r}, t)=\sum_{l=1}^{M} B\left(\mathbf{r}, t, \mathbf{r}_{l}, t_{l}\right)\left(C^{-1}\right)_{k l},
$$

where $C^{-1}$ is the inverse of the $M \times M$ covariance matrix of the data $T_{k}$. When the measurement noise is uniform and uncorrelated, $(C)_{k l}=n \delta_{k l}+B\left(\mathbf{r}_{k}, t_{k}, \mathbf{r}_{l}, t_{l}\right)$ where $\delta_{k j}$ is the Dirac delta and $n$ is the noise variance. The covariance of the error in the estimate $\hat{T}$ is obtained

\footnotetext{
${ }^{2}$ Objective Analysis is also commonly referred to as Optimal Interpolation. It was originally developed by Eliassen et al [14] in 1954 and independently reproduced and popularized by Gandin [15] in 1963.
} 
by direct substitution of (5) and (3) in the integrand of (4) and is given by

$$
\begin{aligned}
& A\left(\mathbf{r}, t, \mathbf{r}^{\prime}, t^{\prime}\right) \triangleq \mathbf{E}[[T(\mathbf{r}, t)-\hat{T}(\mathbf{r}, t)][T(\mathbf{r}, t)-\hat{T}(\mathbf{r}, t)]] \\
& =B\left(\mathbf{r}, t, \mathbf{r}^{\prime}, t^{\prime}\right) \\
& \quad-\sum_{k, l=1}^{M} B\left(\mathbf{r}, t, \mathbf{r}_{k}, t_{k}\right)\left(C^{-1}\right)_{k l} B\left(\mathbf{r}_{l}, t_{l}, \mathbf{r}^{\prime}, t^{\prime}\right) .
\end{aligned}
$$

$A(r, t, r, t)$, the variance of $T$ around the estimate $\hat{T}$, is also known as the a posteriori error. An extensive analysis of the assimilation scheme, equations and generalizations (e.g., multivariate, discrete, non-stationary systems) can be found in [42], [43].

Because estimation errors of a hypothetical sampling array are determined by the statistics of the field noise, $A(\mathbf{r}, t, \mathbf{r}, t)$ can be used as a quantitative measure of the impact of a sequence of measurements on knowledge of the field and allow a priori design of effective sampling arrays [16]. In this paper, the integral of $A(\mathbf{r}, t, \mathbf{r}, t)$ over the domain,

$$
\phi \triangleq \int d \mathbf{r} \int d t A(\mathbf{r}, t, \mathbf{r}, t),
$$

equivalent to (4) evaluated at the optimal $\hat{T}$, is elected as the sampling performance metric to compare and optimize sampling strategies. Substitution of (6) in (7) gives

$$
\begin{aligned}
\phi= & \int d \mathbf{r} \int d t A(\mathbf{r}, t, \mathbf{r}, t) \\
= & \int d \mathbf{r} \int d t(B(\mathbf{r}, t, \mathbf{r}, t) \\
& \left.-\sum_{k, l=1}^{M} B\left(\mathbf{r}, t_{,} \mathbf{r}_{k}, t_{k}\right)\left(C^{-1}\right)_{k l} B\left(\mathbf{r}_{l}, t_{l}, \mathbf{r}, t\right)\right) .
\end{aligned}
$$

Note that this metric depends only on the covariance function $B$ and the data locations and times, $\mathbf{r}_{k}$ and $\mathbf{t}_{k}$.

\section{B. Ocean Statistics}

The coverage metric defined in (8) requires specification of the term, $B\left(\mathbf{r}, t, \mathbf{r}^{\prime}, t^{\prime}\right)$, an estimate of the background statistics. It represents the estimated statistics of the ocean before data assimilation. The diagonal elements $B(\mathbf{r}, t, \mathbf{r}, t)$ describe our confidence in the initial state. The non-diagonal elements represent the covariance between points at different locations and times. They are closely related to the correlation length and the correlation time in the domain [16].

The metric in (8) has a broad range of application and can be used with any positive-definite covariance function $B\left(\mathbf{r}, t, \mathbf{r}^{\prime}, t^{\prime}\right)$. For the purpose of illustrating

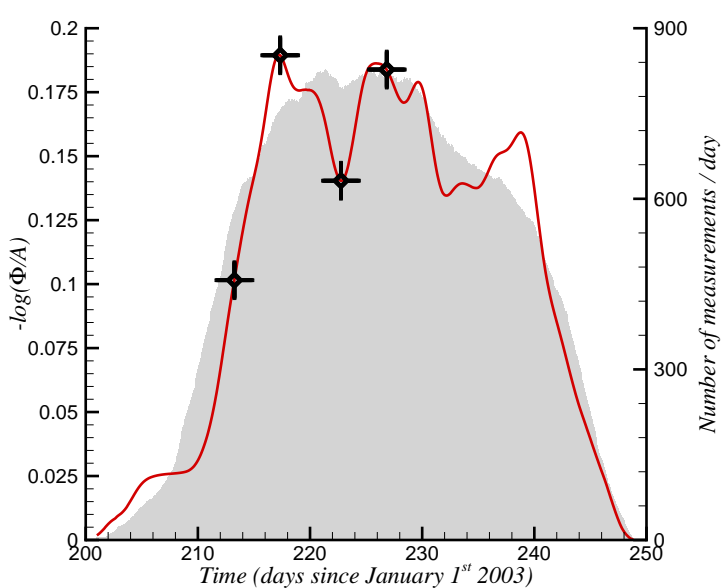

Fig. 6. Sampling metric (solid curve) in units of entropic information and number of profiles (shadowed area) for AOSN 2003. Each cross correspond to a panel of Fig. 5. On August 10th (day 223), the number of profiles is still high but the metric indicates relatively poor coverage. The second panel of Fig. 5 explains this loss of performance by a poor distribution of the gliders in the bay on that day.

the use of the metric, we assume that the background covariance is given by

$$
B\left(\mathbf{r}, t, \mathbf{r}^{\prime}, t^{\prime}\right) \triangleq \sigma_{0} e^{-\frac{\left\|\mathbf{r}-\mathbf{r}^{\prime}\right\|^{2}}{\sigma^{2}}-\frac{\left|t-t^{\prime}\right|^{2}}{\tau^{2}}} .
$$

The parameters $\sigma$ and $\tau$ are the a priori spatial and temperature decorrelation scales; because (9) fixes the structure of the unknown term $B, \sigma$ and $\tau$ can be viewed as inputs to the OA algorithm. In this paper we use $\sigma=$ $25 \mathrm{~km}$ and $\tau=2.5$ days; these values were determined empirically using glider data from Monterey Bay during AOSN 2003 [28]. Notice that the scaling factor $\sigma_{0}$ has no effect on the sampling paths, provided that the measurement noise $n$ is scaled by the same factor. This fact is discussed and exploited in Section VII.

Figure 5 shows a map of the a-posteriori error $A(r, t, r, t)$ at different times during AOSN 2003 where the background covariance is modelled as Gaussian as in (9). The data used correspond to the Spray gliders [22], [28] and the Slocum gliders [13], [28] that patrolled the bay during the summer of 2003 (as plotted in Figures 2 and 3). The metric per unit of time (derivative of (8) with respect to time) is shown on Fig. 6 in units of entropic information [44].

\section{Optimal and Near-Optimal Collectives}

In the context of ocean sampling, not only can (8) be used to quantify the performance of a particular array or formation, but it also provides a means to search for 

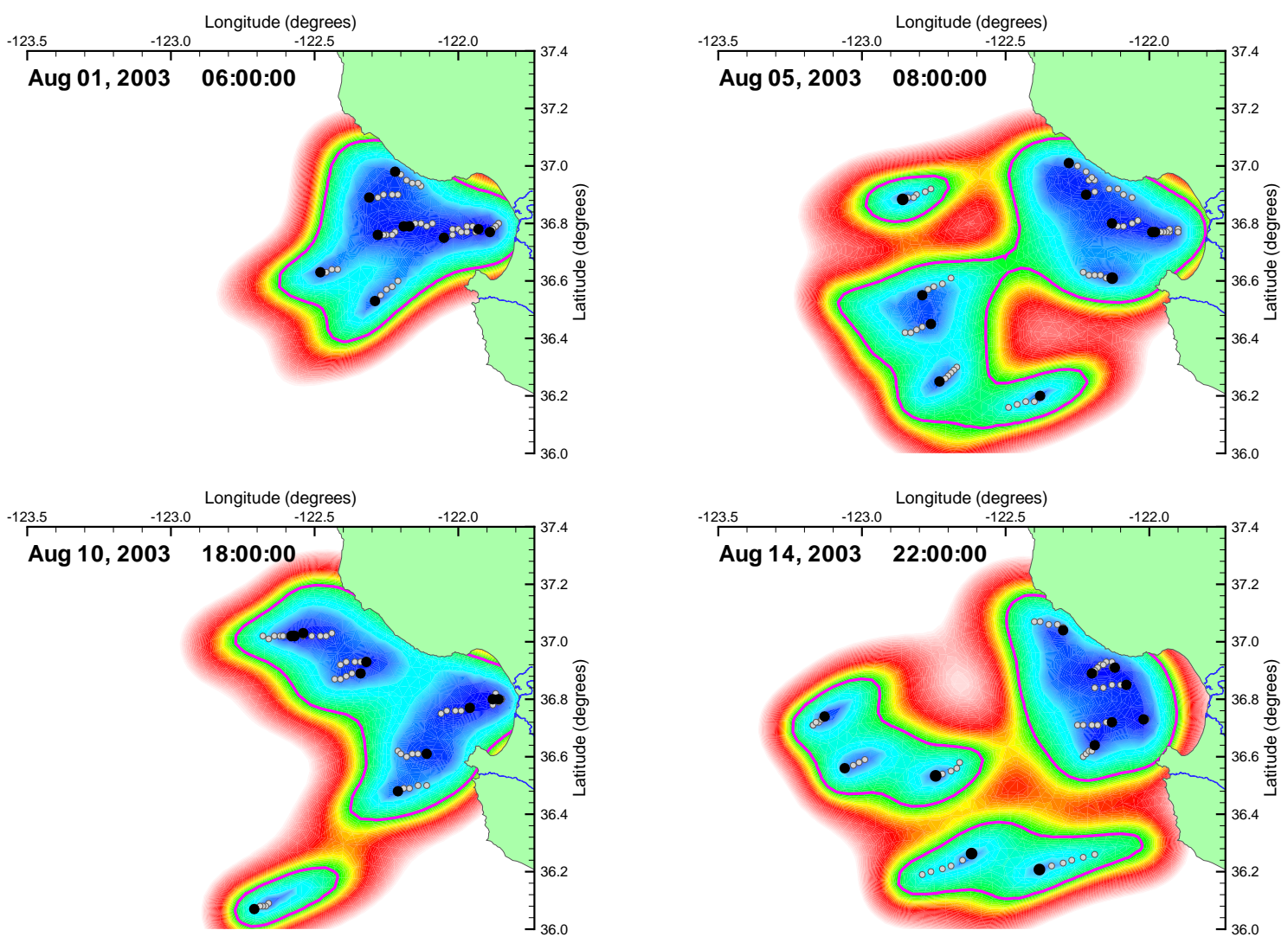

Fig. 5. Error map at different times during the AOSN 2003 experiment. Blue represents small error (good coverage) and red and white represents high error (poor coverage). For each panel, black dots indicates the reported position of the vehicles at the given time. The white dots represent their positions during the last 12 hours. The magenta line encloses all the points where the error has been reduced from its initial state by at least $85 \%$. The sampling metric is shown on Fig 6. Notice that all the gliders are clustered near the coast on August 10th explaining the drop in coverage performance visible on Fig. 6.

optimal sampling strategies. The glider array is viewed as a set of $N$ trajectories $\mathbf{r}_{k}(t)$ satisfying the constraint

$$
\dot{\mathbf{r}}_{k}(t)=\mathbf{v}, k=1, \ldots, N
$$

where $\mathbf{v}$ is velocity relative to the flow and speed $\|\mathbf{v}\|=v$ is fixed. Each glider generates a sequence of measurements $\left(\mathbf{r}_{k}^{l}, t_{l}\right)=\left(\mathbf{r}_{k}(l \Delta t), l \Delta t\right)$, where $\Delta t$ is the sampling period, i.e. the time between profiles. The set of all measurements at a particular depth gathered by the $N$ gliders can be substituted in (8) to determine the performance of the array that we write as $\phi(\overrightarrow{\mathbf{r}})$, where $\overrightarrow{\mathbf{r}}=\left(\mathbf{r}_{1}, \ldots, \mathbf{r}_{N}\right)$. A set of optimal trajectories for these gliders is a set of $N$ curves satisfying (10) and such that $\phi(\overrightarrow{\mathbf{r}})$ is minimum.

Such optimal trajectories are usually complicated and unstructured. In addition, their computation requires a minimization in a large functional space, which may not always be desirable for real-time applications. In this work, instead of optimizing individual trajectories, we consider the optimization of collectives parametrized by a restricted number of parameters. For example, Sections VI and VII focus on arrays of vehicles moving around ellipses. For such trajectories the parameters are the number of ellipses and the number of vehicles per ellipse, the position, size and eccentricity each ellipse as well as the relative position of each pair of vehicles as they move around their ellipses (formulated below as relative phases). Clearly, the computation of the minimum in parametrized families is a much more tractable problem. However, the interest of optimizing the sampling performance over parametrized collectives rather than over individual trajectories extends beyond the numerical convenience. Parametrized collectives are essential to achieve the following:

- Closed-loop control. For each proposed collective, a feedback control is designed that makes it an exponential attractor of the closed-loop dynamics. Feedback control of the collective motion provides robustness for the relative motion of the vehicles in contrast to a decentralized tracking control of each vehicle along its individual reference trajectory.

- Robustness. The robustness of an optimal collective can be studied in terms of the derivatives of the 
metric with respect to the parameters of the family (see Section VI and VII). Small second derivatives indicate flat minima and solutions that are more robust to perturbations such as uncertainty in GPS measurements, deviations due to the flow field or communication problems.

- Interpretation of the data. By restricting the choice of collectives to specific geometries, the data collected along these paths can more easily be interpreted in terms of oceanographic sections [45].

In Section VI, we present the development of coordinated control for gliders on circles and on ellipses. In Section VII, we investigate a parametrized family of elliptical collectives in more detail and determine the optimal collective within this parametrized family.

\section{Parametrization of collectives}

Parametrized families of collectives over closed curves involving the least number of parameters are circles. If we specialize to circles, the optimal parameters to be computed are the number of circles, the number of gliders per circle, the origin and radius of each circle and the relative positions of the gliders on their respective circles. The relative position of two gliders moving around the same circle can be represented by the difference in their headings; this difference is fixed since the gliders move at constant speed. The difference in the headings is equal to the relative phase of the gliders around the circle. To see this suppose the gliders move at unit speed around a single circle of radius $\rho_{0}=\left|\omega_{0}\right|^{-1}$ and centered at the origin. The position of the $k$ th glider at time $t$ is $\mathbf{r}_{k}(t)=\left(\rho_{0} \cos \left(\omega_{0} t+\gamma_{k}\right), \rho_{0} \sin \left(\omega_{0} t+\gamma_{k}\right)\right)$, where $\gamma_{k}$ is the phase of the $k$ th glider. The derivative of $\mathbf{r}_{k}$ with respect to time is $\dot{\mathbf{r}}_{k}=\operatorname{sgn}\left(\omega_{0}\right)\left(\sin \left(\omega_{0} t+\right.\right.$ $\left.\left.\gamma_{k}\right), \cos \left(\omega_{0} t+\gamma_{k}\right)\right)$. The velocity of the glider can also be expressed as $\dot{\mathbf{r}}_{k}=\left(\cos \theta_{k}, \sin \theta_{k}\right)$ where $\theta_{k}$ is the glider's heading angle. By equating these two expressions for $\dot{\mathbf{r}}_{k}$ we get $\omega_{0} t+\gamma_{k}+\operatorname{sgn}\left(\omega_{0}\right) \pi / 2=\theta_{k}$. Thus, the relative heading of two vehicles is equal to their relative phase, i.e., $\theta_{j}-\theta_{k}=\gamma_{j}-\gamma_{k}$. In the top left panel of Figure 7, two vehicles move around circles with $\gamma_{2}-\gamma_{1}=0$. In the top right panel, $\gamma_{2}-\gamma_{1}=\pi$.

Suppose now that two gliders move at unit speed about two different circles, each with radius $\rho_{0}$ but with non-coincident centers. In this case the relative heading (and therefore relative phase) of the two gliders remains constant and the relative position of the gliders is periodic. The periodic function can easily be described by the relative phase and relative position of the circle origins. Let the distance between the circle origins be $d_{0}$. Then, if the relative phase is zero, the gliders are (a)

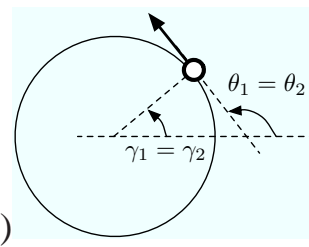

(b)

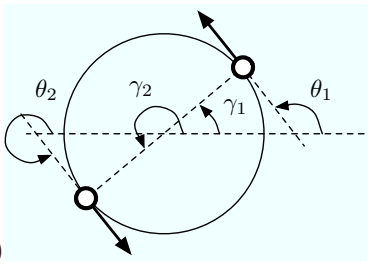

(c)

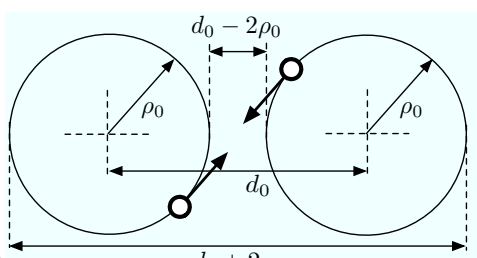

Fig. 7. Cartoons of vehicles moving around closed curves with prescribed relative phases; a) Two vehicles with relative phase equal to zero move around a circle; b) Two vehicles with relative phase equal to $\pi$ move around a circle; c) Two vehicles with relative phase equal to $\pi$ and each vehicle moving around a different circle; d) A closed curve with rotational order of symmetry $L=4$. Four vehicles move around it with fixed relative phase.

synchronized and their relative distance remains constant and equal to $d_{0}$. If the relative phase is $\pi$ then the relative distance of the vehicles varies from its minimum at $d_{0}-2 \rho_{0}$ to its maximum at $d_{0}+2 \rho_{0}$. This is illustrated in the bottom left panel of Figure 7.

Because relative phase is constant for vehicles moving at constant speed around circles of the same radius, we parameterize relative position of a pair of gliders by their relative phase. This makes the stabilizing control problem one of driving vehicles to circles of given radius with prescribed, fixed, relative phases (equivalently, relative headings). For example, suppose $N$ gliders are to move around the same circle. An example of an optimal solution in a homogeneous field is one in which the gliders are uniformly distributed around the circle (called the splay state formation). This is equivalent to phase locking with relative phase between neighboring gliders equal to $2 \pi / N$, which we study in the next section.

Relative phase can be useful as a prescription of relative position even for closed curves of more general shape. The choices of relative phase that can be kept constant for constant speed vehicles moving around a given shape depend on the rotational order of symmetry of the shape. The rotational order of symmetry of a shape is equal to $L \in \mathbb{N}_{0}$ if the shape looks unchanged after it is rotated about its center by angle $2 \pi / L$. For example, a hexagon has rotational symmetry of order six, a square has symmetry of order four, a rectangle and an ellipse have symmetry of order two. A shape with rotational order of symmetry equal to one has no rotational symmetry.

Consider a shape with rotational order of symmetry 
equal to $L$. If we choose the relative phase for a pair of gliders moving at constant speed around the shape to be an integer multiple of $2 \pi / L$, the relative phase will remain constant. An example for $L=4$ is shown in Figure 7. In the case of circles, as discussed above, any relative phase can be selected. In the case of ellipses, only two choices of relative phase can be selected; these are either relative phase equal to zero or equal to $\pi$, when the gliders are synchronized or anti-synchronized, respectively, as they move around a single ellipse or up to $N$ identical ellipses with non-coincident centers.

In Section VI we describe steering control laws for stabilization of gliders to circles and ellipses with phase locking.

\section{CoOrdinated Control}

This section describes feedback control laws that stabilize collective motion of a planar model of autonomous vehicles moving at constant speed. Following Section V, we consider vehicles moving around closed curves with given, fixed relative phases. As described in Section V-D, relative phases determine, in part, the relative positions of the vehicles. In the case of collective motion around circles of equal radius, the relative phase is identical to relative heading and is also constant. For more general shapes, prescribed relative phases are chosen as an integer multiple of $2 \pi / L$ where $L$ is the rotational order of symmetry of the shape. For example, in the case of coordinated motion of gliders around ellipses, $L=2$ and we design stabilizing controllers that fix relative phases to 0 or $\pi$.

Each glider is modeled as a point mass with unit mass, unit speed and steering control. We first provide a feedback control law that stabilizes circular motion of the group of vehicles about its center of mass. This control law depends on the relative position of the vehicles. Next, we address the problem of stabilizing the relative phases of the circling vehicles. An additional control term, depending only on the relative headings of the vehicles, stabilizes symmetric patterns of the vehicles in the circular formation.

As long as the feedback control is a function only of the relative positions and headings of the vehicles, the system is invariant to rigid rotation and translation in the plane. This corresponds to the symmetry group, $S E(2)=\mathbb{R}^{2} \otimes S O(2) \equiv \mathbb{R}^{2} \otimes S^{1}$, where $\otimes$ is the semi-direct product. We show how breaking this symmetry can lead to useful variations on circular formations. First, we introduce a fixed beacon to break the $\mathbb{R}^{2}$ symmetry. Second, we introduce a reference heading which breaks the $S^{1}$ symmetry. In addition, we introduce interconnection topologies for the spacing and orientation coupling which stabilize collective motion of coordinated subgroups of vehicles. This includes the case in which there are multiple circles with a different subgroup of vehicles moving around each circle.

Finally, we describe a control law to stabilize collective motion on more general shapes. More specifically, we stabilize a single vehicle on an elliptical trajectory about a fixed beacon. Additionally, we couple vehicles on separate ellipses using their relative headings in order to synchronize the vehicle phases about each ellipse.

\section{A. Circular Control}

The vehicle model that we study is composed of $N$ identical point-mass vehicles subject to planar steering control. The vehicle model is

$$
\begin{aligned}
\dot{\mathbf{r}}_{k} & =v e^{i \theta_{k}} \\
\dot{\theta}_{k} & =u_{k}, \quad k=1, \ldots N
\end{aligned}
$$

where $\mathbf{r}_{k}=x_{k}+i y_{k} \in \mathbb{C} \equiv \mathbb{R}^{2}$ and $\theta_{k} \in S^{1}$ are the position and heading of each vehicle, $v$ is the vehicle speed relative to the flow, and $u_{k}$ is the steering control input to the $k$ th vehicle. In this section, we assume unit vehicle speed, i.e. $v=1$, and ignore the flow. In Section V, the position $\mathbf{r}_{k}$ of the $k$ th vehicle was a vector in $\mathbb{R}^{2}$.

In this section, we exploit the isometry between $\mathbb{R}^{2}$ and $\mathbb{C}$ and we view $\mathbf{r}_{k}$ as an element of the real $^{3}$ vector space $\mathbb{C}$. The real vector spaces $\mathbb{C}$ and $\mathbb{C}^{N}$ give us more flexibility in choosing an inner product ${ }^{4}$. We define the inner product by

$$
\left\langle\mathbf{z}_{1}, \mathbf{z}_{2}\right\rangle=\operatorname{Re}\left\{\overline{\mathbf{z}}_{1}^{\top} \mathbf{z}_{2}\right\},
$$

where $\overline{\mathbf{z}}_{1}^{T}$ represents the conjugate transpose of $\mathbf{z}_{1}$ and $\operatorname{Re}\{\cdot\}$ is the real part of a complex number. We view $\mathbf{z}_{1}$ and $\mathbf{z}_{2}$ as the elements of the real vector space $\mathbb{C}^{N}$ (i.e., isomorphic to $\mathbb{R}^{2 N}$ ), for which (12) is a valid inner product.

For the sake of brevity, we often stack identical variables for each vehicle in a common vector. For example, $\vec{\theta}=\left(\theta_{1}, \ldots \theta_{N}\right) \in T^{N}$ contains all the headings and $\overrightarrow{\mathbf{r}}=\left(\mathbf{r}_{1}, \ldots \mathbf{r}_{N}\right) \in \mathbb{C}^{N}$ contains all the positions.

To help understand the model (11), consider the following two examples of constant control input. For

\footnotetext{
${ }^{3}$ By real vector space, we mean a vector space for which the field of scalars is $\mathbb{R}$. Complex vector spaces are defined with complex scalars. For example, $\mathbb{C}^{N}$ is both a real and a complex vector space. In this paper, we consider $\mathbb{C}^{N}$ as a real vector space only.

${ }^{4}\left\langle\mathbf{z}_{1}, \mathbf{z}_{2}\right\rangle=\operatorname{Re}\left\{\overline{\mathbf{z}}_{1}^{\top} \mathbf{z}_{2}\right\}$ is not an inner product for the complex vector spaces $\mathbb{C}$ because it violates sesquilinearity. However, it is a valid inner product for the real vector spaces $\mathbb{C}$ and $\mathbb{C}^{N}$.
} 
$u_{k}=\omega_{0} \in \mathbb{R}_{0}$, the vehicles travel on fixed circles of radius $\rho_{0}=\left|\omega_{0}\right|^{-1}$. The sense of rotation is given by the sign of $\omega_{0}$. For $u_{k}=\omega_{0}=0$, each vehicle follows a straight trajectory in the direction of the initial heading.

Due to the unit speed and unit mass assumptions we can relate the coherence of vehicle headings to the motion of the group. Let the center of mass of the group be $\mathbf{R}=\frac{1}{N} \sum_{j=1}^{N} \mathbf{r}_{j}$. Also, let the order parameter $\mathbf{p}_{\vec{\theta}} \in \mathbb{C}$, denote the centroid of the vehicle headings on the unit circle in the complex plane. The order parameter is equivalent to the velocity of the center of mass of the group, i.e.

$$
\mathbf{p}_{\vec{\theta}}=\frac{1}{N} \sum_{k=1}^{N} e^{i \theta_{k}}=\frac{1}{N} \sum_{k=1}^{N} \dot{\mathbf{r}}_{k}=\dot{\mathbf{R}} .
$$

Notice that we have $\left|\mathbf{p}_{\vec{\theta}}\right| \leq 1$. We define a potential function $U_{1}$ by

$$
U_{1}(\vec{\theta})=\frac{N}{2}\left|\mathbf{p}_{\vec{\theta}}\right|^{2} .
$$

The gradient of $U_{1}$ is given by

$$
\frac{\partial U_{1}}{\partial \theta_{k}}=\left\langle i e^{i \theta_{k}}, \mathbf{p}_{\vec{\theta}}\right\rangle, k=1, \ldots, N .
$$

Notice that certain distinguished motion of the group correspond to critical points of $U_{1}$. For instance, $U_{1}(\vec{\theta})$ is maximum for parallel motion of the group $(\forall k$ : $\left.\theta_{k}=\theta_{0}\right)$ and minimum when the center of mass is fixed $\left(\mathbf{p}_{\vec{\theta}}=\dot{\mathbf{R}}=0\right)$. We refer to solutions for which $\mathbf{p}_{\vec{\theta}}=\dot{\mathbf{R}}=0$ as balanced solutions since the headings are distributed around the unit circle in such a (balanced) way that the center of mass of the group is fixed. Letting $\overrightarrow{1}=(1, \cdots, 1) \in \mathbb{R}^{N}$, we use (14) to observe that $\left\langle\nabla U_{1}, \overrightarrow{1}\right\rangle=\left\langle i \mathbf{p}_{\vec{\theta}}, \mathbf{p}_{\vec{\theta}}\right\rangle=0$; this corresponds to the $S^{1}$ rotational symmetry of the system since $U_{1}$ is invariant to rigid rotation of the vehicle headings.

To stabilize circular motion of the group about its center of mass, we introduce a dissipative control law that is a function of the relative positions $\mathbf{r}_{k j}=\mathbf{r}_{k}-\mathbf{r}_{j}$. Let the vector from the center of mass to vehicle $k$ be $\tilde{\mathbf{r}}_{k}=\mathbf{r}_{k}-\mathbf{R}=\frac{1}{N} \sum_{j=1}^{N} \mathbf{r}_{k j}$. We propose to control the vehicles using

$$
u_{k}=\omega_{0}\left(1+\kappa\left\langle\tilde{\mathbf{r}}_{k}, \dot{\mathbf{r}}_{k}\right\rangle\right), \quad k=1, \ldots N
$$

where $\kappa>0$ is a scalar gain. For intuition regarding the control law (15), note that for $\kappa=0$, vehicle $k$ will undergo circular motion with radius $\left|\omega_{0}\right|^{-1}$ and direction of rotation determined by the sign of $\omega_{0}$. The gain $\kappa$ regulates the contribution to the control of a dissipation term which drives vehicle $k$ such that its velocity is perpendicular to the vector from the center of mass of the group. The dissipation term evaluates to zero for circular motion around a fixed center of mass.

The stability of the circular motion of the group about a common point can be studied using standard Lyapunov functions. Consider the function

$$
S(\overrightarrow{\mathbf{r}}, \vec{\theta})=\frac{1}{2} \sum_{k=1}^{N}\left|e^{i \theta_{k}}-i \omega_{0} \tilde{\mathbf{r}}_{k}\right|^{2}, \quad \omega_{0} \neq 0,
$$

which has minimum zero for circular motion around the center of mass with radius $\rho_{0}=\left|\omega_{0}\right|^{-1}$ and direction of rotation determined by the sign of $\omega_{0}$. Differentiating $S(\overrightarrow{\mathbf{r}}, \vec{\theta})$ along the solutions of the vehicle model gives

$$
\dot{S}=\sum_{k=1}^{N}\left\langle\omega_{0} \tilde{\mathbf{r}}_{k}, \dot{\mathbf{r}}_{k}\right\rangle\left(\omega_{0}-u_{k}\right) .
$$

Therefore, using the circular control (15), we find that

$$
\dot{S}=-\kappa \sum_{k=1}^{N}\left\langle\omega_{0} \tilde{\mathbf{r}}_{k}, \dot{\mathbf{r}}_{k}\right\rangle^{2} \leq 0,
$$

and $S$ is an acceptable Lyapunov function for this system. Consequently, solutions converge to the largest invariant set, $\Lambda$, for which $\dot{S}=0$. This yields the following result.

Theorem 6.1: Consider the vehicle model (11) with the circular control (15). All solutions converge to a circular formation of radius $\rho_{0}=\left|\omega_{0}\right|^{-1}$. Moreover, the relative headings converge to an arrangement that is a critical point of the potential $U_{1}(\vec{\theta})$. In particular, balanced circular formations form an asymptotically stable set of relative equilibria.

The technical details of the proof can be found in [46]. Notice that solutions in $\Lambda$ have the dynamics $\dot{\vec{\theta}}=\omega_{0} \overrightarrow{1}$, i.e. vehicles follow circles of radius $\left|\omega_{0}\right|^{-1}$. The set of balanced circular solutions for which all circles are coincident corresponds to the minimum of the potential $S(\overrightarrow{\mathbf{r}}, \vec{\theta})$. Simulations suggest that this set of equilibria has almost global convergence.

\section{B. Control of Relative Headings}

If, in addition to the relative positions, we feed back the relative headings of the vehicles, we can stabilize particular phase-locked patterns or arrangements of the vehicles in their circular formation. Let the potential $U(\vec{\theta})$ satisfy $\langle\nabla U, \overrightarrow{1}\rangle=0$ so that it is invariant to rigid rotation of all the vehicle headings. We combine the circular control (15) with a gradient control term as follows:

$$
u_{k}=\omega_{0}\left(1+\kappa\left\langle\tilde{\mathbf{r}}_{k}, \dot{\mathbf{r}}_{k}\right)\right\rangle-\frac{\partial U}{\partial \theta_{k}} .
$$


The circular motion of the group in a phase-locked heading arrangement is a critical point of $U(\theta)$. The stability of the motion can be proved by showing the existence of a Lyapunov function. For instance take,

$$
V(\overrightarrow{\mathbf{r}}, \vec{\theta})=\kappa S(\overrightarrow{\mathbf{r}}, \vec{\theta})+U(\vec{\theta}),
$$

where $S(\overrightarrow{\mathbf{r}}, \vec{\theta})$ is defined in (16). The time derivative of $V(\overrightarrow{\mathbf{r}}, \vec{\theta})$ along the solutions of the vehicle dynamics is given by

$$
\dot{V}=\sum_{k=1}^{N}\left(\kappa\left\langle\omega_{0} \tilde{\mathbf{r}}_{k}, \dot{\mathbf{r}}_{k}\right\rangle-\frac{\partial U}{\partial \theta_{k}}\right)\left(\omega_{0}-u_{k}\right) .
$$

Substitution of the composite control (17) in (19) gives

$$
\dot{V}=-\sum_{k=1}^{N}\left(\kappa\left\langle\omega_{0} \tilde{\mathbf{r}}_{k}, \dot{\mathbf{r}}_{k}\right\rangle-\frac{\partial U}{\partial \theta_{k}}\right)^{2} \leq 0 .
$$

Therefore, solutions converge to the largest invariant set, $\Lambda$, for which $\dot{V}=0$. A detailed proof can be found in [46] and yields the following theorem

Theorem 6.2: Consider the vehicle model (11) and a smooth heading potential $U(\theta)$ that satisfies $\langle\nabla U, \overrightarrow{1}\rangle=$ 0 . The control law (17) enforces convergence of all solutions to a circular formation of radius $\rho_{0}=\left|\omega_{0}\right|^{-1}$. Moreover, the relative headings converge to an arrangement that is a critical point of the potential $\kappa U_{1}+U$. In particular, every minimum of $U$ for which $U_{1}=0$ defines an asymptotically stable set of relative equilibria.

This result enables us to stabilize symmetric patterns of the vehicles in circular formations. Symmetric $(M, N)$-patterns of vehicles are characterized by $2 \leq$ $M \leq N$ heading clusters separated by a multiple of $\frac{2 \pi}{M}$. Figure 8 depicts the six possible different symmetric phase patterns for $N=12$ and is not meant to imply that the vehicles are collocated, rather that their velocity phasors may be. There is a one-to-one correspondence between these symmetric patterns and global minima of specifically designed potentials [46]. In order to define these potentials, we extend the notion of the order parameter of vehicle headings to include higher harmonics, i.e.

$$
\mathbf{p}_{m \vec{\theta}}=\frac{1}{m N} \sum_{k=1}^{N} e^{i m \theta_{k}} .
$$

The objective is to consider potentials of the form

$$
U_{m}(\vec{\theta})=\frac{N}{2}\left|\mathbf{p}_{m \vec{\theta}}\right|^{2},
$$

which satisfy $\left\langle\nabla U_{m}, \overrightarrow{1}\right\rangle=0$. These potentials are used to prove the following [46]:

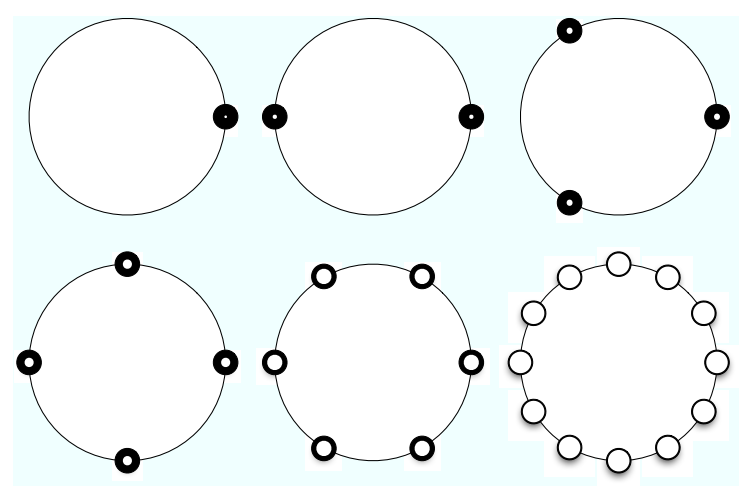

Fig. 8. The six possible different symmetric patterns for $N=$ 12 corresponding to $M=1,2,3,4,6$ and 12 . The top left is the synchronized state and the bottom right is the splay state. The number of collocated headings is illustrated by the width of the black annulus denoting each phase cluster.

Lemma 6.1: Let $1 \leq M \leq N$ be a divisor of $N$. Then $\vec{\theta} \in T^{N}$ is an $(M, N)$-pattern if and only if it is a global minimum of the potential

$$
U^{M, N}=\sum_{m=1}^{\left\lfloor\frac{N}{2}\right\rfloor} K_{m} U_{m}
$$

where $\left\lfloor\frac{N}{2}\right\rfloor$ is the largest integer less than or equal to $\frac{N}{2}$ and $K_{m}$ are arbitrary coefficients satisfying

$$
\begin{cases}K_{m}<0 & \text { if } \frac{m}{M} \in \mathbb{N}, \\ K_{m}>0 & \text { otherwise. }\end{cases}
$$

Theorem 6.2 together with Proposition 6.1 yield a prescription for stabilizing symmetric patterns. Of particular interest for mobile sensor networks is stabilizing the circular formation in which the vehicles are evenly spaced, i.e. the $(N, N)$-pattern or splay state formation [47]. This formation is characterized by $\mathbf{p}_{m \vec{\theta}}=0$ for $m=1, \ldots N-1$ and $\left|\mathbf{p}_{N \vec{\theta}}\right|=\frac{1}{N}$. Consequently, we define the splay state potential to be

$$
U^{N, N}=K \sum_{m=1}^{\left\lfloor\frac{N}{2}\right\rfloor} U_{m}, K>0 .
$$

The splay state formation control law has the form (17) with $U(\vec{\theta})$ given by (20) and can be written

$$
u_{k}=\omega_{0}\left(1+\kappa\left\langle\tilde{\mathbf{r}}_{k}, \dot{\mathbf{r}}_{k}\right\rangle\right)+\frac{K}{N} \sum_{j=1}^{N\lfloor N / 2\rfloor} \sum_{m=1}^{\left\lfloor\sin m \theta_{k j}\right.} \frac{}{m} .
$$

A simulation of the splay state formation for $N=12$ vehicles is shown in Figure 9. Twelve vehicles start from random initial conditions and the controller (21) enforces convergence to a circular orbit with uniform spacing (i.e., the phase difference between adjacent vehicles is $\frac{2 \pi}{12}$ ). 


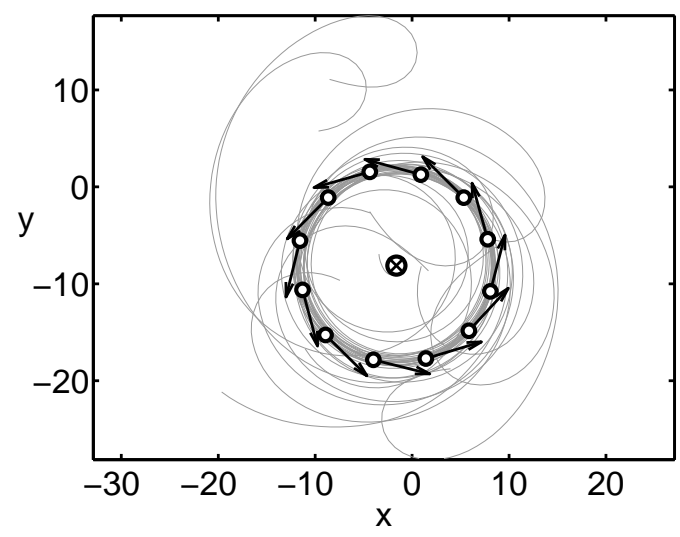

Fig. 9. A numerical simulation of the splay state formation starting from random initial conditions using the control (21) with $N=12$, $\omega_{0}=0.1, \kappa=\omega_{0}$ and $K=\omega_{0}^{2}$. Each vehicle and its velocity is illustrated by a black circle and an arrow. Note that the center of mass of the group, illustrated by a crossed circle, is fixed at steady-state.

\section{Planar Symmetry Breaking}

The feedback control laws in sections VI-A and VI-B require only the relative positions and headings of the vehicles and, consequently, they are invariant to rigid translation and rotation in the plane. This corresponds to the symmetry group, $S E(2)=\mathbb{R}^{2} \otimes S^{1}$. In this section, we introduce variations of these control laws which break the translation and rotation symmetries. First, we break the $\mathbb{R}^{2}$ translation symmetry by stabilizing the circular formation about a fixed beacon. Secondly, we break the $S^{1}$ rotational symmetry by coupling the vehicles to a heading reference.

The position of the fixed beacon is referred to as $\mathbf{R}_{0} \in \mathbb{C}$. The relative position from the beacon is defined as $\tilde{\mathbf{r}}_{k}=\mathbf{r}_{k}-\mathbf{R}_{0}$. A formal proof uses the Lyapunov function $S(\overrightarrow{\mathbf{r}}, \vec{\theta})$ defined in (16) with the new definition of $\tilde{\mathbf{r}}_{k}$. Furthermore, Theorem 6.2 continues to hold for circular motion about the fixed beacon [46]. That is, the control (17) can be used to stabilize circular motion to the set of heading arrangements that are critical points of the potential $U(\vec{\theta})$, where $\langle\nabla U, \overrightarrow{1}\rangle=0$. Clearly, this applies to the splay state potential (20).

Next, we introduce a heading reference $\theta_{0}$ where $\dot{\theta}_{0}=$ $\omega_{0}$. Let $u_{k}, k=1, \ldots, N-1$ be given by (17) where $U(\vec{\theta})$ is a potential that satisfies $\langle\nabla U, \overrightarrow{1}\rangle=0$. The $N$ th vehicle is coupled to the heading reference using

$$
u_{N}=\omega_{0}\left(1+\kappa\left(\tilde{\mathbf{r}}_{k}, \dot{\mathbf{r}}_{k}\right)\right)-\frac{\partial U}{\partial \theta_{k}}+d \sin \left(\theta_{0}-\theta_{N}\right),
$$

where $d>0$. Critical points of $U(\vec{\theta})$ that satisfy $\theta_{N}=\theta_{0}$ define an asymptotically stable set [46]. To prove this result, we use the composite Lyapunov function

$$
W(\overrightarrow{\mathbf{r}}, \vec{\theta})=V(\overrightarrow{\mathbf{r}}, \vec{\theta})+d\left(1-\cos \left(\theta_{0}-\theta_{N}\right)\right)
$$

where $V(\overrightarrow{\mathbf{r}}, \vec{\theta})$ is given by (18). The complete analysis can be found in [46]. The set of circular formations that minimizes $U(\vec{\theta})$ and satisfy $\theta_{N}=\theta_{0}$ are the global minima of $W(\overrightarrow{\mathbf{r}}, \vec{\theta})$. For $\omega_{0}=0$, the control (22) can be used to track piecewise linear trajectories [48].

\section{Coordinated Subgroups}

In this section, we design control laws to coordinate vehicles in subgroups using block all-to-all interconnection topologies. Here, the term "block" refers to a subgroup of vehicles and "all-to-all" refers to the interconnection topology of that subgroup. It is assumed that the subgroups are not interconnected unless otherwise stated. In other words, the vehicles can be distributed among subgroups, each subgroup corresponding to vehicles moving on a different circle or ellipse. First, we introduce a block all-to-all interconnection topology for the circular control term that depends on the relative positions. This restriction on the coupling yields stability of subgroups of vehicles in separate circular formations. Similarly, block all-to-all coupling applied to the gradient control term that depends on relative headings yields heading arrangements within subgroups of vehicles. We illustrate the use of block all-to-all couplings on a scenario of practical interest. The vehicles are divided into three subgroups that minimize the splay state potential such that each subgroup is in a splay state formation.

We refer to each vehicle subgroup by its block index $b=1, \ldots, B$, where $B$ is the total number of blocks. Let $N^{b}$ be the number of vehicles in block $b$. Note that $N^{b} \geq 2$ except in the case of fixed beacons in which $N^{b} \geq 1$.

We assume that each vehicle is assigned to one and only one block, so that $\sum_{b=1}^{B} N^{b}=N$. Also, let $F^{b}=$ $\left\{f_{1}^{b}, \ldots, f_{N^{b}}^{b}\right\}$ be the set of vehicles indices in block $B$. The center of mass of block $b$ is given by

$$
\mathbf{R}^{b}=\frac{1}{N^{b}} \sum_{k=1}^{N^{b}} \mathbf{r}_{f_{k}^{b}}
$$

Similarly, the $m$-th moment of the heading distribution of block $b$ is

$$
\mathbf{p}_{m \vec{\theta}}^{b}=\frac{1}{m N^{b}} \sum_{k=1}^{N^{b}} e^{i m \theta_{f_{k}^{b}}}, \quad m=1,2, \ldots .
$$

Using (23), we can also define block-specific heading potentials such as

$$
U_{m}^{b}(\vec{\theta})=\frac{1}{2}\left|\mathbf{p}_{m \vec{\theta}}^{b}\right|^{2}
$$


Note that $\frac{\partial\left(U_{m}^{b}\right)}{\partial \theta_{k}}=0$ for $k \notin F^{b}$ and $\left\langle\nabla U_{m}^{b}, \overrightarrow{1}\right\rangle=0$.

Using this notation, we summarize the following corollaries to Theorems 6.1 and 6.2 [46]. First, consider block all-to-all coupling for the circular control term only. In this case, the control law (17) with $\tilde{\mathbf{r}}_{k}=\mathbf{r}_{k}-\mathbf{R}^{b}$ and $k \in F^{b}$ enforces convergence of all solutions to circular formations of radius $\rho_{0}=\left|\omega_{0}\right|^{-1}$ in phase arrangements that are critical points of the potential $\kappa U_{1}^{b}+U$ as in Theorem 6.2, where $U(\vec{\theta})$ is a potential that satisfies $\langle\nabla U, \overrightarrow{1}\rangle=0$. In particular, the circular motion of all the vehicles in a block have coincident centers. Alternatively, suppose we use block all-to-all coupling only in the gradient control term that depends on relative headings. In this case, the control law is (17), where $U(\vec{\theta})=\sum_{b=1}^{B} U^{b}(\vec{\theta})$ and $U^{b}(\vec{\theta})$ is a potential depending only on the headings in block $b$ that satisfies $\left\langle\nabla U^{b}, \overrightarrow{1}\right\rangle=0$. This control enforces convergence of all solutions to circular formations of radius $\rho_{0}=\left|\omega_{0}\right|^{-1}$ in phase arrangements that are critical points of the potentials $\kappa U_{1}+U^{b}$.

To demonstrate the use of the control law (17), we present the result of a useful case of block all-to-all spacing coupling with fixed beacons. In this example, the phase coupling is both all-to-all and block all-to-all with

$$
U=U^{(N, N)}+\sum_{b=1}^{B} U^{b\left(N^{b}, N^{b}\right)}
$$

where $N^{b}=N / B$ for $b=1, \ldots, B$ and is $U^{b\left(N^{b}, N^{b}\right)}$ is given by (20). This potential is minimized by the phase arrangement in which the entire group, as well as each block, are in the splay state of vehicle headings. In other words, if all the vehicle heading phasors were plotted on the same unit circle, then the resulting pattern would be the splay state for $N$ headings (see Figure (8). Taken separately, the headings phasors for block $b$ are also in the splay state for $N^{b}$ headings. Simulation results for $N=12$ and $B=3$ are shown in Figure 10. The twelve vehicles start from random initial positions and organize themselves in the splay states using (17).

\section{E. Shape Control: Elliptical Beacon Control Law}

In this section, we modify the circular control law and we stabilize a single vehicle on an elliptical trajectory about a fixed beacon. We use a generalization of the potential $S(\overrightarrow{\mathbf{r}}, \vec{\theta})$ in (16) to prove Lyapunov stability of this trajectory. Additionally, we couple several vehicles via their relative headings as in Section VI-B in order to synchronize the vehicle phases on each ellipse.

Let $\mathbf{R}_{0} \in \mathbb{C}$ and $\mu_{0} \in S^{1}$ represent the center and orientation of an ellipse with the lengths of the semi-

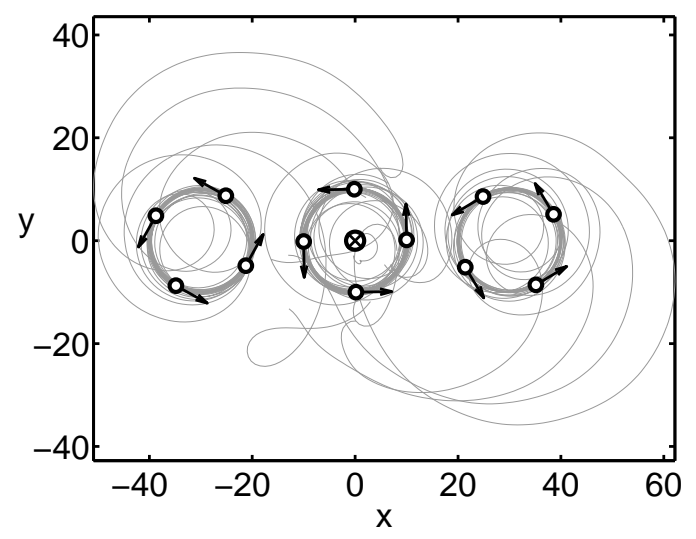

Fig. 10. Simulation results for $N=12$ and $B=3$ starting from random initial conditions with block all-to-all spacing coupling and three fixed beacons at $\left(\mathbf{R}_{0}^{1}, \mathbf{R}_{0}^{2}, \mathbf{R}_{0}^{3}\right)=(-30,0,30)$. Phase coupling is all-to-all and block all-to-all with the potential (25). The simulation parameters are $\kappa=\omega_{0}=1 / 10$.

(a)

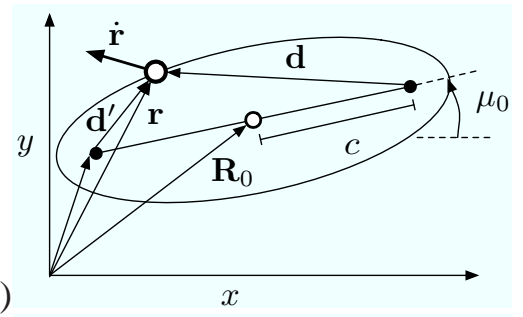

(b)

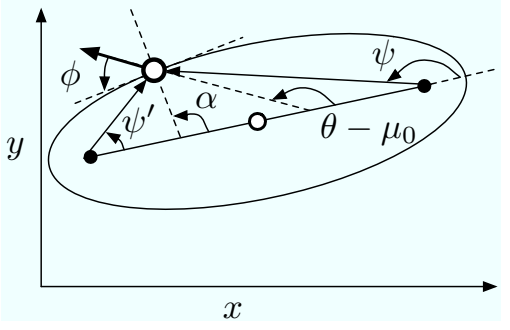

Fig. 11. a) The vectors $\mathbf{d}$ and $\mathbf{d}^{\prime}$ used to identify the position of the vehicle (white circles) relative to the focii (black circles) for an ellipse centered at $\mathbf{R}_{0}$ and rotated by $\mu_{0}$. b) Depicts the angles $\psi$, $\psi^{\prime}, \alpha$, and $\phi$ used in the control design. Note that $\phi=0$ for stable elliptical motion with positive rotation.

major and -minor axes given by $a$ and $b$. The positions of the focii are $\mathbf{R}_{0} \pm c e^{i \mu_{0}}$, where $c=\sqrt{a^{2}-b^{2}}$. Let $\mathbf{d} \in \mathbb{C}$ and $\mathbf{d}^{\prime} \in \mathbb{C}$ be the relative positions of the vehicle from each focus, defined by

$$
\begin{gathered}
\mathbf{d} \triangleq \rho e^{i\left(\psi+\mu_{0}\right)}=\mathbf{r}-\mathbf{R}_{0}-c e^{i \mu_{0}} \\
\mathbf{d}^{\prime} \triangleq \rho^{\prime} e^{i\left(\psi^{\prime}+\mu_{0}\right)}=\mathbf{r}-\mathbf{R}_{0}+c e^{i \mu_{0}}
\end{gathered}
$$

and shown in Panel (a) of Figure 11.

For a single vehicle whose position and heading are $\mathbf{r}$ and $\theta$, respectively, motion along the ellipse is characterized by

$$
\frac{\rho+\rho^{\prime}}{2}=a
$$


and

$$
\frac{\psi+\psi^{\prime}}{2}=\theta-\mu_{0} \pm \frac{\pi}{2} .
$$

Condition (28) requires that the average distance to the focii remains constant. Condition (29) requires that the average angular position measured from both focii must be separated by $\pm \frac{\pi}{2}$ from the angle made by the velocity vector and the major axis. Notice that the term $\pm \frac{\pi}{2}$ corresponds to either clockwise or counterclockwise motion around the ellipse. Condition (29) is a straightforward consequence of the following property of ellipses [49] p. 3: "if a source of light is located at one of the foci of a mirror having the form of an ellipse, the reflected light will converge at the other focus."

For vehicles moving continuously in the plane, $\psi$ and $\psi^{\prime}$ are continuous functions. Therefore, the average $(\psi+$ $\left.\psi^{\prime}\right) / 2$ in condition (29) is moving on only one branch (clockwise or counter-clockwise) and can never switch continuously from one branch to the other. The physical interpretation of this property is the following: a vehicle moving along an ellipse with a constant speed cannot change its sense of rotation and keep a continuous motion. Without loss of generality, we will only consider the positive (counter-clockwise) branch of condition (29). Building on these geometrical considerations, we define the shape coordinates $(\xi, \eta, \alpha, \beta, \phi)$ given by

$$
\begin{aligned}
\xi & =\frac{\rho+\rho^{\prime}}{2} \\
\eta & =\frac{\rho-\rho^{\prime}}{2} \\
\alpha & =\frac{\psi+\psi^{\prime}}{2} \\
\beta & =\frac{\psi-\psi^{\prime}}{2} \\
\phi & =\alpha+\frac{\pi}{2}-\theta+\mu_{0} .
\end{aligned}
$$

The angles $\alpha$ and $\phi$ are shown in Figure 11.b. In these coordinates, the conditions for elliptical motion (28) and (29) are equivalent to $(\xi, \phi)=(a, 0)$ and $\dot{\xi}=\dot{\phi}=0$. We choose the Lyapunov function candidate

$$
S(\xi, \eta, \alpha, \beta, \phi)=\frac{1}{2}\left|\xi-a e^{-i \phi}\right|^{2}
$$

which has minimum at zero for an elliptical trajectory centered at $\mathbf{R}_{0}$ and rotated by $\mu_{0}$ with major and minor semi-axes $(a, b)$.

The time derivative of the Lyapunov function (35) along the trajectories of (53)-(57) (see appendix) is

$$
\begin{aligned}
\dot{S} & =\left(\xi-a e^{-i \phi}, \dot{\xi}+i a e^{-i \phi} \dot{\phi}\right) \\
& =(\xi-a \cos \phi) \dot{\xi}+\xi a \sin \phi(\dot{\alpha}-u) .
\end{aligned}
$$

The dynamics of the single vehicle in the shape coordinates are derived in the Appendix. Using these calculations and choosing the control, $u$, with scalar gain, $\kappa>0$,

$$
u=\dot{\alpha}+\kappa \frac{\xi}{a} \sin \phi+\frac{1}{\xi a}(\xi-a \cos \phi) \cos \beta
$$

gives

$$
\dot{S}=-\kappa \xi^{2} \sin ^{2} \phi \leq 0 .
$$

Note that for the circular case $a=b=\left|\omega_{o}\right|^{-1}$, the control reduces to the circular beacon control law (15), which can be expressed in the shape coordinates $(\rho, \phi)$ as

$$
u=\omega_{0}(1+\kappa \rho \sin \phi) .
$$

We obtain the following result:

Theorem 6.3: Almost all trajectories of (11) for a single vehicle subject to the control (36) converge to an elliptical trajectory centered at $\mathbf{R}_{0}$ and rotated by $\mu_{0}$. The size of the ellipse is parameterized by the length of its semi-major and -minor axes, $a$ and $b$.

Proof: By the Lasalle invariance principle, all trajectories converge to the largest invariant set for which $\dot{S}=0$. Using (37), the invariance condition becomes $\sin \phi=0$ since $\xi>0$. Subject to this condition, the dynamics of the shape variables $(\xi, \phi)$ from equations (53) and (57) in the appendix become

$$
\begin{aligned}
\dot{\xi} & =0 \\
\dot{\phi} & =-\frac{1}{\xi a}(\xi-a \cos \phi) \cos \beta .
\end{aligned}
$$

Setting equations (38) and (39) equal to zero, we obtain the solutions $(\xi, \phi)=(a, 0)$ and $\sin \phi=\cos \beta=0$. The latter corresponds to trajectories on the major axis of the ellipse (between the focii) and does not constitute an invariant set due to the singularities in (36) at the focii. As a result, all trajectories which do not originate at a focus of the ellipse asymptotically converge to the set for which $(\xi, \phi)=(a, 0)$. This set corresponds to elliptical motion with parameters $(a, b)$.

We briefly discuss how to extend this result to coordinate groups of vehicles on (separate) ellipses by coupling their headings as in Section VI-B. Let $\mathbf{R}_{0}^{1}, \ldots, \mathbf{R}_{0}^{N}$ and $\mu_{0}^{1}, \ldots, \mu_{0}^{N}$ be the location and orientation of $N$ ellipses with parameters $\left(a_{k}, b_{k}\right)$. Also, let $u_{k}^{e_{k}}$ be the ellipse control (36) corresponding to the $k$ th ellipse. We assume that the ellipses' circumferences are all the same. Then, in order to stabilize each vehicle to its ellipse and to synchronize the phases of all the vehicles, we propose the control

$$
u_{k}=u_{k}^{e_{k}}+K \frac{\partial U_{1}}{\partial \theta_{k}}, k=1, \ldots, N,
$$


for $K>0$, where $U_{1}$ is the potential function (13). The convergence analysis of this control law is not pursued in the present paper but simulations suggest good convergence properties. In Section VII, we compute the optimal sampling ellipses for a group of two gliders. The optimal ellipses have the same circumferences and the controller derived here is applied to this case (see Fig. 16).

\section{Optimal Coordinated Solutions}

In this section, we use the sampling metric defined by (8) to compute near-optimal vehicle trajectories constrained to ellipses. The objective of this section is to determine the optimal ellipse parameters as a function of the size, shape and characteristic scales of the region of interest and the capabilities of the sensor platforms. We start by introducing a convenient formalization of the adaptive sampling problem using non-dimensional parameters. Next, we present the results of numerical optimization experiments for a single vehicle on an elliptical trajectory and for a pair of vehicles on separate ellipses. Lastly, we consider the influence of a uniform flow field on the sampling performance of the ellipse feedback control from the previous section. We anticipate that the insights from these numerical results will extend to larger groups of vehicles.

\section{A. Sampling Numbers}

We consider a rectangular domain $\mathcal{B}$ of size $\mathcal{B}_{a} \times \mathcal{B}_{b}$ in which we would like optimal sensor coverage during a finite duration of time $\mathcal{T}$. The trajectories of the $N$ vehicles, given by $\mathbf{r}_{k}(t)$, and the sampling metric, $\phi(\overrightarrow{\mathbf{r}})$ (see (8)), determine the locations and effectiveness of the sensor measurements, respectively. The optimal trajectories, $\mathbf{r}_{k}^{*}(t)$, and the value of the metric at the optimum, $\phi^{*} \triangleq \phi\left(\overrightarrow{\mathbf{r}}^{*}\right)$, are obtained by minimizing the metric $\phi$ among all acceptable sets of curves, $\mathbf{r}_{k}$, $k=1, \ldots, N$, satisfying the constant velocity constraint in (10).

We decrease the number of dimensions of the optimization problem by applying the Buckingham $\pi$ theorem [50] to reduce the number of parameters. Let $\operatorname{Av}\{X\}$ represent the space-time average of a quantity $X$ over the domain $\mathcal{B} \times[0, \mathcal{T}]$. Then the initial uncertainty on the field, $\sigma_{0}$, is given by

$$
\sigma_{0}=\operatorname{Av}\{B(\mathbf{r}, t, \mathbf{r}, t)\} .
$$

Recall from Section V-A, the measurement noise is denoted by $n$. We now define precisely the correlation

\begin{tabular}{|c|l||c|c|c|}
\hline Name & Description & Dist. & Time & Temp. \\
\hline \hline$\sigma_{0}$ & Initial Uncertainty, (9), (41) & 0 & 0 & 1 \\
$\sigma$ & Correlation Length, (9), (42) & 1 & 0 & 0 \\
$\tau$ & Correlation Time, (9), (43) & 0 & 1 & 0 \\
\hline$n$ & Measurement Noise & 0 & 0 & 1 \\
\hline$v$ & Speed of Sensors & 1 & -1 & 0 \\
\hline $\mathcal{B}_{a}$ & Width of Domain & 1 & 0 & 0 \\
$\mathcal{B}_{b}$ & Height of Domain & 1 & 0 & 0 \\
$\mathcal{T}$ & Duration of Experiment & 0 & 1 & 0 \\
\hline \hline $\mathfrak{r}_{k}^{*}(t)$ & Optimal Traj. for kth Veh. & 1 & 0 & 0 \\
$\phi^{*}$ & Minimum Metric & 2 & 1 & 2 \\
\hline
\end{tabular}

\section{TABLE I}

RELEVANT PHYSICAL QUANTITIES AND THEIR DIMENSIONS. DIMENSION OF QUANTITY IS THE PRODUCT OF DISTANCE, TIME AND TEMPERATURE, EACH TO THE POWER DEFINED BY THE CORRESPONDING ENTRY.

length and the correlation time by

$$
\begin{aligned}
& \sigma \triangleq \frac{1}{\pi} \operatorname{Av}\left\{\int_{\mathbb{R}^{2}} d \mathbf{r}^{\prime} \frac{B\left(\mathbf{r}, t, \mathbf{r}^{\prime}, t\right)}{B(\mathbf{r}, t, \mathbf{r}, t)}\right\} \\
& \tau \triangleq \frac{1}{\sqrt{\pi}} \operatorname{Av}\left\{\int_{-\infty}^{+\infty} d t^{\prime} \frac{B\left(\mathbf{r}, t, \mathbf{r}, t^{\prime}\right)}{B(\mathbf{r}, t, \mathbf{r}, t)}\right\} .
\end{aligned}
$$

One can easily check that, for a Gaussian correlation function given by (9), the equations above are equivalent to the usual definition of the correlation length and time. For a Gaussian covariance model, the definitions (42) and (43) give the $1 / e$ decorrelation scale (i.e., the distance and time at which the correlation reaches $1 / e \approx 37 \%$ ).

The advantage of (42) and (43) is that they extend the definition to arbitrary correlation function $B$. Notice that, for practical application, the integrals cannot be taken over an infinite domain (in space and time). The domain of interest is usually finite and $B\left(\mathbf{r}, t, \mathbf{r}^{\prime}, t^{\prime}\right)$ is not defined outside this domain. In practice, the correlation function becomes quickly negligible when $\left\|\mathbf{r}-\mathbf{r}^{\prime}\right\|$ or $\left|t-t^{\prime}\right|$ grows, therefore, integrating over a finite spatial domain $\mathcal{B}$ and finite interval of time $[0, \mathcal{T}]$ gives essentially the same result as (42) and (43).

We assume that the shape of the stochastic component of the field (see [51], [52] for details) is well captured by the correlation length and the correlation time defined in (42) and (43).

Table I lists the eight relevant variables and their respective dimensions. We use temperature as a proxy for the (arbitrary) units of the sensor measurements. Since we are looking for the minimum value of the metric, we add the variable $\phi^{*}$ to the first eight variables. The rank of the matrix made by the units of this system is 3 (see Table I). According to the Buckingham $\pi$ theorem 
[53], the relationship giving the $\phi^{*}$ can be reduced to a relationship between 6 non-dimensional numbers. For practical reasons, the following choices of these numbers will be used in this work:

- $\Phi=\phi^{*} / \sigma_{0} \mathcal{B}_{a} \mathcal{B}_{b} \mathcal{T}$, the normalized metric;

and the sampling numbers,

- $\mathrm{S} \mathfrak{z}=\sqrt{\mathcal{B}_{a} \mathcal{B}_{b}} / \sigma$, the size of the domain,

- $\mathrm{Sh}=\mathcal{B}_{b} / \mathcal{B}_{a}$, the shape of the domain,

- $\mathrm{St}=\mathcal{T} / \tau$, the sampling time interval,

- $\mathrm{Sp}=v \tau / \sigma$, the normalized speed of the vehicle,

- $\mathrm{Sn}=n / \sigma_{0}$, the sensor noise.

A similar development for the optimal trajectories leads to the definition of the scaled optimal trajectories

$$
\mathfrak{r}_{k}^{*}(s)=\frac{1}{\mathcal{B}_{a}} \mathbf{r}_{k}^{*}(\tau t), k=1, \ldots, N .
$$

where $s=\tau t$ is the normalized time variable. The constant speed constraint (relative to the flow) in (10) translates into

$$
\|\mathbf{v}\|=\left\|\frac{d \mathfrak{r}_{k}^{*}}{d s}\right\|=\frac{v \tau}{\mathcal{B}_{a}}=\frac{\mathrm{Sp}}{\mathrm{S}_{\mathfrak{z}}} \sqrt{\mathrm{Sh}} .
$$

Notice that the use of $S \mathfrak{z}$ and $S \mathfrak{h}$ allows us to study the system in terms of its size (the area of the box is $\sigma^{2} \mathrm{Sz}^{2}$ ) and its aspect ratio (shape). Both $\mathrm{Sp}$ and $\mathrm{Sn}$ can be fixed or limited to a small range for a specific experiment with a homogeneous group of vehicles and sensors. During an experiment, the survey speed of the sensor platforms, $v$, is typically known and fixed and the characteristic spatial/temporal scales can be estimated. For example, during the AOSN 2003 experiment, the effective glider speed, $v$, (including surface intervals) was between 25 and $35 \mathrm{~cm} / \mathrm{s}$. The glider data was used to approximate the average correlation length, $\sigma \approx 25 \mathrm{~km}$, and time, $\tau \approx$ 2.5 days (see [28] for details). Therefore, the sampling number $\mathrm{Sp}$ was between 2 and 3 for this experiment. Similarly, Sn only depends on the sensor noise and the a-priori uncertainty of the model.

In the remainder of this paper, we will only consider experiments that last much longer than the characteristic time scale. In other words, we assume that $\mathcal{T} \gg \tau$ or, equivalently, St $\gg 1$. For the AOSN experiment, the estimated correlation time was 2.5 days (see [28]). The gliders sampled the region for about a month, so $\mathrm{St} \approx$ 12 , which is sufficiently high to validate our analysis. For $\mathcal{T} \gg 1$, one expects to get the same normalized performance for any interval of time $\mathcal{T}$. In other words, we assume that the metric per unit of area and time, $\Phi$, is independent of the sampling time, St. We summarize the functional dependence of the normalized performance metric on the four remaining sampling numbers by

$$
\Phi=\Phi(\mathrm{S} \mathfrak{z}, \mathrm{Sh}, \mathrm{Sp}, \mathrm{Sn}) .
$$

In the next subsection, we compute the near-optimal trajectories of a single vehicle among a family of ellipses. These racetracks can be pre-computed or, alternatively, optimized in real-time to maximize the steady-state performance of the array. The feedback control presented in Section VI is essential to maintain the vehicles on these optimal tracks in the presence of strong currents and communication difficulties.

\section{B. Optimal Ellipses in Rectangular Domains}

In this subsection, we present optimization results for a single vehicle following a parameterized elliptical trajectory in a rectangular domain. The objective is to find the set of parameters yielding the smallest value of the metric, (45), as a function of the sampling numbers. A system with only one sensor moving on an elliptical path has six degrees of freedom: the position and orientation of the ellipse, the lengths of the semi-major and -minor axes $a$ and $b$, and the initial phase, $\gamma(0)$. One can easily check that these six parameters determine a unique trajectory for the vehicle (up to the sense of rotation).

Inspection of (8) directly reveals that the center of the optimal ellipse necessarily coincides with the middle of the box $\mathcal{B}$. Moreover, the angle $\gamma(0)$ has no influence on the metric for $\mathrm{St} \gg 1$ and can be ignored. In addition, we assume that the ellipse orientation $\mu_{0}$ is parallel to the long side of the box.

For given size $S_{\mathfrak{z}}$, shape $\mathrm{Sh}$, sensor noise $\mathrm{Sn}$ and relative vehicle speed $S \mathfrak{p}$, the problem reduces to a twodimensional space where the variables are the lengths of the semi-major and -minor axes of the ellipse, $a$ and $b$. For example, Figure 12 shows the contour levels of the metric, i.e. the error map, as a function of $a$ and $b$ for the sampling numbers $S_{\mathfrak{z}}=2, S \mathfrak{h}=1, S \mathfrak{n}=0.1$ and $\mathrm{Sp}=3$. There is a unique minimum for a vehicle moving on a circle of radius $a=b=0.256$. The fact that the optimal ellipse is a circle is consistent with the square shape of the domain.

Also notice that the minimum in Figure 12 is relatively "flat". Small deviations from a prescribed optimal plan do not have much influence on the metric; this suggests robustness to disturbances such as strong currents and intermittent feedback. The error map associated with this optimal trajectory is shown in the upper left panel of Figure 13. Next, we investigate the influence of each sampling number on the optimal elliptical solution.

1) Independence of the Shape Sh: In Figure 14, we plot the performance of optimal elliptical trajectories for a single vehicle within the rectangular box $\mathcal{B}$ as a function of the sampling numbers $S \mathfrak{z}$ and $S \mathfrak{h}$. The shape of the optimal trajectory varies with the shape of the 


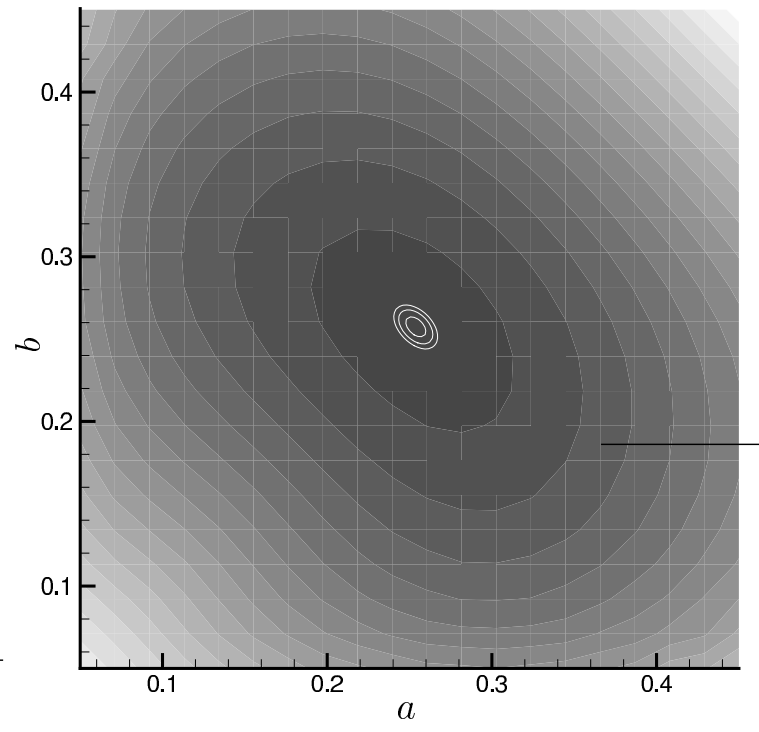

Fig. 12. Non-dimensional metric $\Phi$ for one vehicle on an elliptical trajectory with semi-major and -minor axis lengths $a$ and $b$. The gray scale is proportional to the value of $\Phi$, from low uncertainty (dark) to high (light). The sampling numbers are $S_{\mathfrak{z}}=2, \mathrm{Sh}=1$, $\mathrm{S} \mathfrak{n}=0.1, \mathrm{~S} \mathfrak{p}=3$. The minimum gives the near optimal ellipse (a circle) $a=b=0.256$.
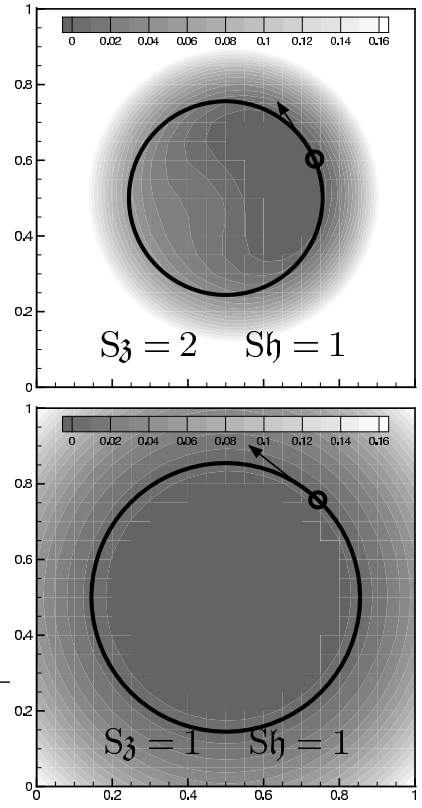

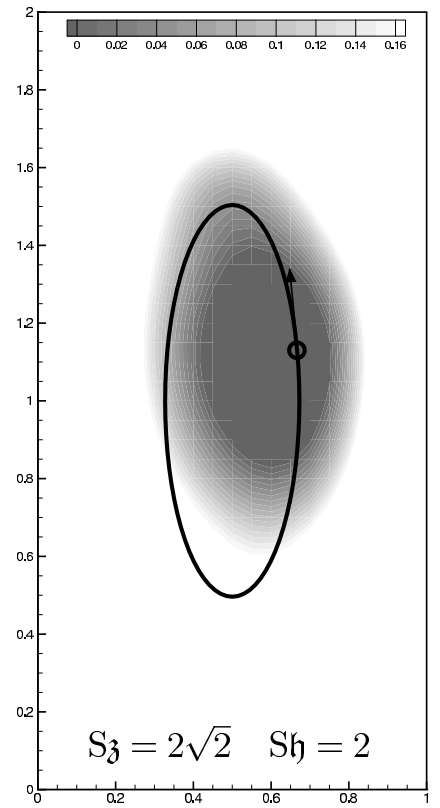

Fig. 13. Snapshots in time of the error maps associated with the near optimal elliptical trajectories for selected values of the parameters. $\mathrm{Sn}=0.1$ and $\mathrm{Sp}=3$. Vehicle position is represented by a small circle and velocity by a vector.

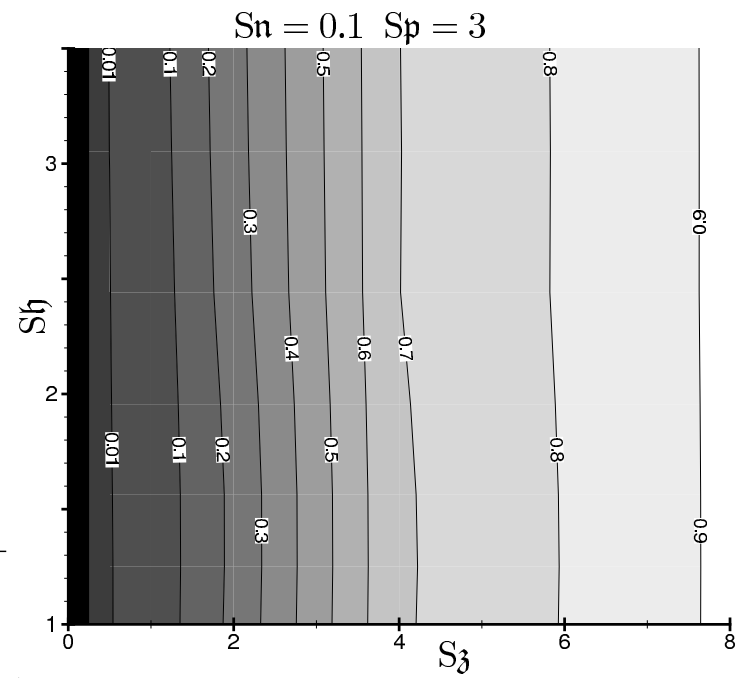

Fig. 14. Optimal value of metric $\Phi$ as a function of $S_{\mathfrak{z}}$ and $S \mathfrak{h}$ for $\mathrm{Sn}=0.1$ and $\mathrm{Sp}=3$ with a single vehicle on an elliptical trajectory. The elliptical trajectory at each point yields the minimal value of $\Phi$ for the corresponding values of $S_{\mathfrak{z}}$ and $S \mathfrak{h}$. The gray scale is proportional to the value of $\Phi$, from low uncertainty (dark) to high (light). Within numerical accuracy, $\Phi$ is independent of $S \mathfrak{h}$, the shape of the domain. The plot shows that the same performance can be achieved on a rectangle of any aspect ratio (with the appropriate optimal trajectory that will vary with shape).

domain; however, the contour levels of $\Phi$ in the $\left(\mathrm{S}_{\mathfrak{z}}, \mathrm{Sh}\right)$ plane reveal that $\Phi$ does not depend on $\mathrm{Sh}$. As a result, the same performance can be achieved on rectangles with different aspect ratio but with the same area. In particular, if a complex domain such as Monterey Bay is divided in several sub-regions patrolled by groups of gliders, the shape of the sub-regions can be chosen freely. This permits a greater flexibility in designing sampling plans.

2) Role of Speed $\mathrm{Sp}$ and Noise $\mathrm{Sn}$ : To study the influence of the sampling numbers $S \mathfrak{p}, S \mathfrak{n}$ and $S \mathfrak{z}$ on the optimal trajectories, the optimal ellipses and the minimum value of the metric are computed for several values of the sampling numbers. For example, see Figure 13 for typical error maps. We have already determined that the shape $S \mathfrak{h}$ and the time number $\mathrm{St}$ do not influence the solutions so we present results for $\mathrm{Sh}=1$ and $\mathrm{St} \gg 1$. Figure 15 gives the optimal non-dimensionalized radius $(a=b)$ and the minimum value of the metric, $\Phi$, as a function of $\mathrm{S}_{\mathfrak{z}}$. Each curve corresponds to different values of $S \mathfrak{n}$ and $S \mathfrak{p}$. Notice that, for $S \mathfrak{z}>S \mathfrak{p}, \Phi$ becomes independent of $\mathrm{Sn}$.

Figure 15 also shows that $\mathrm{Sp}$ has no influence on the performance metric (although it does determine the perimeter of the optimal trajectory). The minimum value of $\Phi$ is determined entirely by the noise $S \mathfrak{n}$ and the size of the domain $\mathrm{S} \mathfrak{z}$. On the other hand, the optimal 

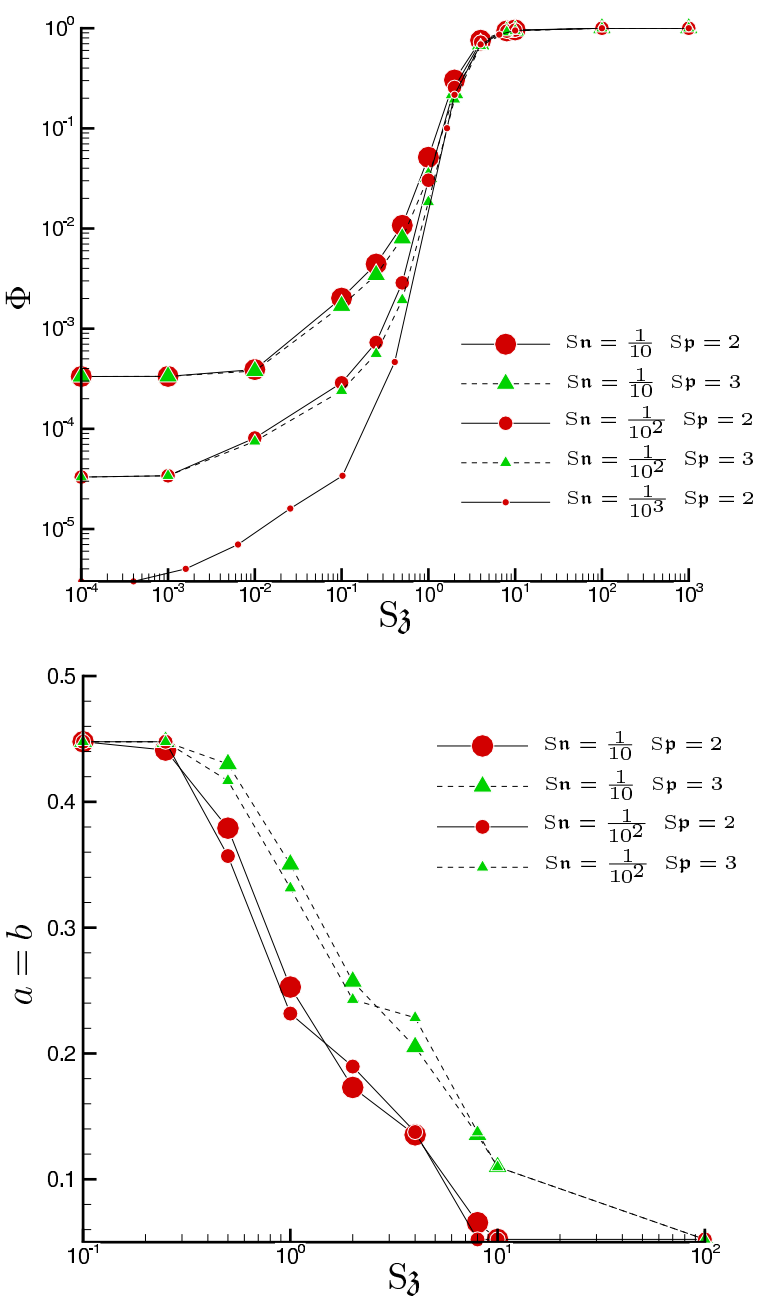

Fig. 15. Top Panel: Value of the metric for the optimal circular trajectory of one vehicle as a function of $\mathrm{S}_{\mathfrak{z}}$. Bottom Panel: Radius of the optimal circle as a function of $\mathrm{S} \mathfrak{z}$. Each curve correspond to different values of the sensor noise $S \mathfrak{n}$ and the vehicle speed $\mathrm{Sp}$. Notice that $\Phi$ does not depend on Sp. Moreover, the optimal radius does not depend on $\mathrm{Sn}$.

trajectory (i.e., the radius of the circle) is a function of $\mathrm{S} z_{\mathfrak{z}}$ and $\mathrm{Sp}$, but does not depend on the measurement noise $\mathrm{Sn}$. This is an important result that allows us to design optimal trajectories independently of the precision of the sensors.

\section{Multiple Vehicle Results}

In this section, we study the optimal elliptical trajectories for two vehicles in a square spatial domain. We also consider the influence of the flow field on the ellipse feedback control from Section VI using the performance metric. We assign the sampling numbers $S_{\mathfrak{z}}=1$ and $\mathrm{Sh}=1$ in order to simplify analysis of the results. We use the feedback control to simulate the vehicle trajectories on the optimal ellipses. The top panels of

\begin{tabular}{|c|c|c|c|}
\hline Sim & Flow direction & Heading Coupling & Metric \\
\hline$\# 1$ & $N / A$ & on & 0.018 \\
$\# 2$ & $0^{\circ}$ & on & 0.020 \\
$\# 3$ & $90^{\circ}$ & on & 0.054 \\
$\# 4$ & $180^{\circ}$ & on & 0.023 \\
$\# 5$ & $270^{\circ}$ & on & 0.101 \\
$\# 6$ & $N / A$ & off & 0.236 \\
\hline
\end{tabular}

TABLE II

METRIC FOR SIMULATED OPTIMAL TRAJECTORIES.

The metric is the integral of the squared error over the domain, so the trajectories with a smaller value of the metric are more desirable.

Figure 16 show these trajectories and snapshots of the resulting error map.

For these sampling numbers, the coverage metric is minimized for two ellipses that are (nearly) centered along the horizontal axis. The optimal relative phase difference between the vehicles is zero, i.e. they are synchronized. The vehicles remain synchronized despite the fact that the optimal ellipses have different eccentricities because they have the same perimeter ${ }^{5}$. Any shift in the respective position of the vehicles (e.g., delay or current impeding one vehicle) decreases the performance of the coverage metric [54]. Notice that, in the absence of inhomogeneities and currents, there are four equivalent solutions corresponding to the two ellipses of Figure 16 and the same ellipses rotated by 90,180 and 270 degrees.

1) Influence of Flow Field: To study the robustness of the solution, we used the controller designed in Section VI to stabilize the vehicles to the optimal ellipses in the presence of currents. Table II summarizes these experiments with the magnitude of the flow speed equal to $2 \%$ of the vehicle speed ${ }^{6}$. The path of the vehicles converging toward their optimal ellipses can be seen on the left panels of Figure 16. The corresponding error maps are shown in the right panels of Figure 16.

Comparing simulations \#2 and \#4 in Table II, we observe that currents in the longitudinal direction (i.e., aligned with the major axis of the ellipses) have a very small effect on the performance. On the other

\footnotetext{
${ }^{5} \mathrm{We}$ attribute the $1.4 \%$ difference in the optimal ellipse perimeters to numerical errors in the computation of the metric as well as to the finite optimization time (i.e., the solution may not have completely converged). For the numerical simulation of the ellipse control law, we perturbed the four optimal ellipse parameters $\left(a_{1}, b_{1}, a_{2}, b_{2}\right)$ in order to more precisely match their perimeters without any appreciable degradation of the performance metric.

${ }^{6}$ We limited the flow speed to $2 \%$ because larger magnitude flow velocity significantly distorted the vehicle trajectories due to singularities in the ellipse control law which occur when the vehicle passes near a focus of the ellipse. This is a deficiency in the controller which needs to be addressed in future work.
} 

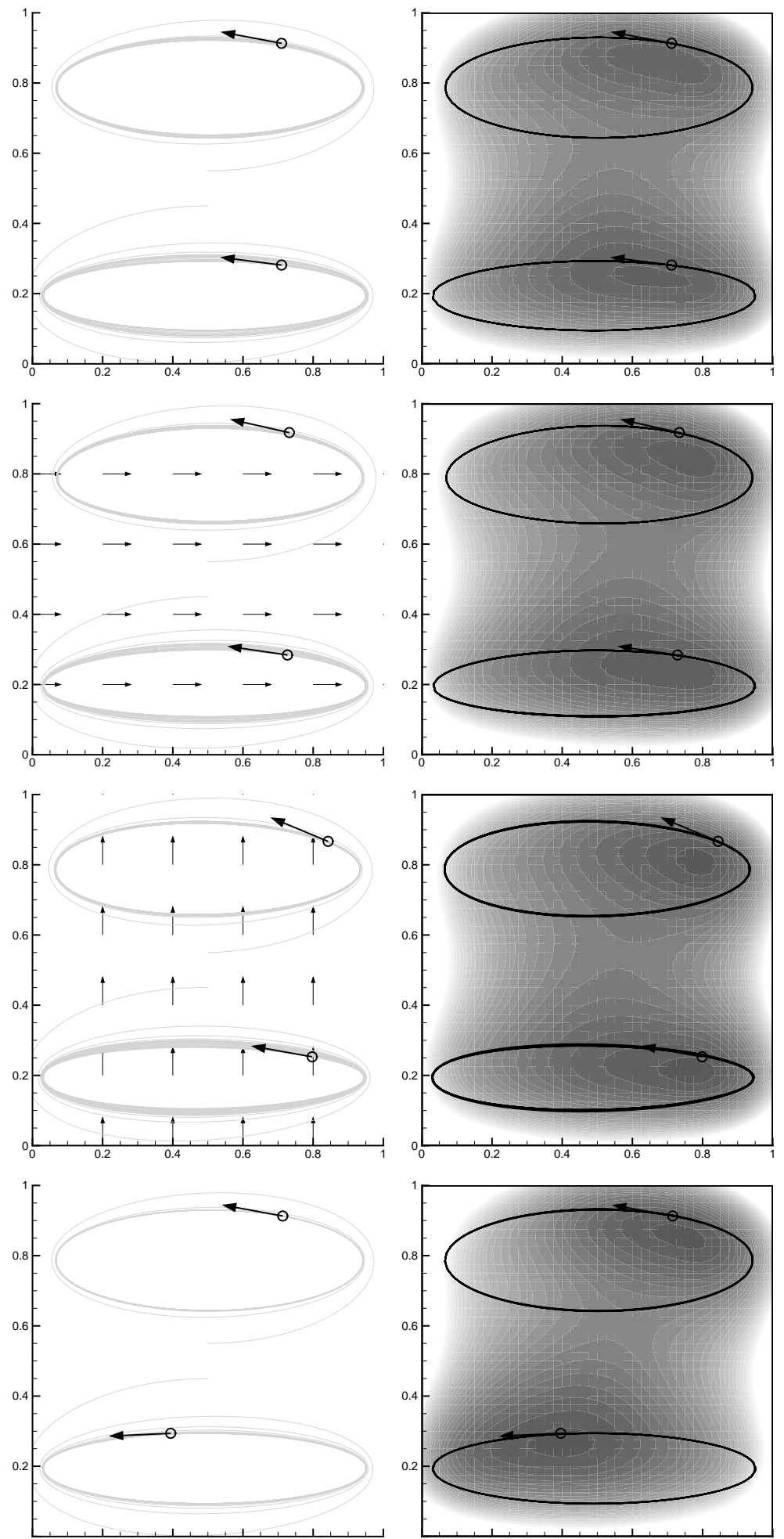

Fig. 16. Optimal ellipse trajectories for two vehicles in a square domain with $\mathrm{S} \mathfrak{z}=1$. The left column shows the simulated trajectories using the feedback control from Section VI to stabilize the vehicles to the optimal ellipses with the control gains $\kappa_{k}=1 / a_{k}$ and $K=0.05$, where $a_{k}$ is the semi-major axis of the $k$ th ellipse for $k=1,2$. The right column shows the resulting error maps for the steady-state measurement distribution. The rows represent simulations \#1, \#2, \#3, \#6 (see Table II). The small circles and heavy vectors show the positions and velocities, respectively, of the vehicles at the time shown. The light arrows represent the direction (and not magnitude) of the flow, if present in the simulation. 
hand, transverse currents have a dramatic effect on the sampling metric. This result contradicts the intuitive result that high eccentricity vehicle trajectories should not be aligned with the prevailing currents.

2) Role of Heading Synchronization: Clearly, the ability of the controller to maintain the "synchronization" of the vehicles is, in large part, responsible for the performance achieved by simulations \#1, \#2 and \#4. To demonstrate the influence of the synchronization, a simulation was run without the heading coupling described in Section VI. The performance for such an array is dramatically worse than the synchronized case. Table II shows that, without heading coupling, the network of vehicles performs even worse in the absence of currents than the synchronized array in the presence of currents.

\section{FINAL REMARKS}

We present developments on the design of mobile sensor networks that optimize sampling performance defined in terms of uncertainty in an estimate of a sampled field over a fixed area. The general problem that we pose, and thus, the methodology that we develop, pertains to mobile sensor networks in a number of domains: land, air, space and underwater.

We address a number of general issues as well as some of the particular issues that distinguish mobile sensor networks in the ocean. For example, we make our solutions robust to strong currents that can push around slow moving mobile sensors by determining optimal solutions in the presence of currents, choosing solutions with performance robust to small deviations and designing feedback control to stably coordinate vehicles.

We determine optimal, coordinated trajectories of mobile sensors over a parametrized family of trajectories. This family consists of multiple closed curves (we specialize to ellipses), each with multiple sensors moving at constant speed. The relative positions of the sensors on these curves are parametrized by relative phases. This low-dimensional parametrization simplifies the optimization problem and motivates the coordinated feedback control laws that include terms modeled after coupled phase oscillator dynamics.

We present optimal solutions in several cases. For example, two sensors, each moving around a different ellipse, are optimized when their phases are synchronized. Sampling performance is significantly enhanced for the closed-loop system with the coordinating feedback control enabled. In the presence of a constant flow field, the solution (with feedback control) with the major axes of the two ellipses aligned with the flow provides higher performance than in the case the flow is aligned with the minor axes of the ellipses.
In related work we are investigating inhomogeneous statistics and alternative methods for computing and adapting the sampling metric. We are developing methodology to further treat and exploit the flow field, to address a range of scales in the sampled field of interest and to make use of a heterogeneous sensor network. We are also investigating how well the data set that optimizes the coverage metric presented in this paper serves the needs of specific high resolution ocean forecasting models.

We describe in the paper a number of practical and critical challenges of operating mobile sensor networks in the ocean: limitations on communication, computing and control, including inherent asynchronicities and latencies. We discuss how we have handled these challenges in previous field work. However, these and other problems related to time and energy optimality remain outstanding open problems of great interest.

Up until recently, our focus has been the optimal design for Eulerian data assimilation. Recent developments in data assimilation extend this concept to Lagrangian data assimilation [55], [56]. In a Lagrangian assimilation scheme, the paths of passive tracers or drifters (as opposed to an estimate of the Eulerian velocity) are assimilated directly into the ocean model. Although it was developed for float data [57], [56], Lagrangian data assimilation represents an exciting application for quasiLagrangian (i.e., weakly propelled) gliders. In particular, a Lagrangian metric and corresponding optimal trajectories could be substituted into the usual objective analysis scheme.

\section{ACKNOWLEDGMENTS}

This paper has greatly benefited from many enlightening discussions with Pierre Lermusiaux (Harvard University), Eddie Fiorelli, Pradeep Bhatta, Fumin Zhang and Spring Berman (Princeton University) and Ralf Bachmayer (National Research Council of Canada).

Figure 4 was provided by Eddie Fiorelli, Pradeep Bhatta and Ralf Bachmayer. The authors are grateful to Eddie Fiorelli and Fumin Zhang (Princeton University) for sharing their latests advances in formation design and control.

This research was funded in part by Office of Naval Research grants N0014-02-1-0826 and N00014-02-10861 and N00014-04-1-0534 and in part by the Belgian Program on Inter-university Poles of Attraction, initiated by the Belgian State, Prime Minister's Office for Science, Technology and Culture. Derek Paley was supported by a National Science Foundation Graduate Research Fellowship, the Princeton Gordon Wu Graduate Fellowship and the Pew Charitable Trust Grant 2000-002558. 


\section{APPENDIX}

\section{SHAPE DYNAMICS FOR ELLIPTICAL CONTROL}

We first derive the dynamics of a single vehicle in the coordinates $\left(\rho, \rho^{\prime}, \psi, \psi^{\prime}, \theta\right)$. Differentiating the definitions (26) and (27) using $\dot{\mathbf{R}}_{0}=\dot{\mu}_{0}=0$ and applying the model (11) for a single vehicle gives

$$
\begin{aligned}
\dot{\mathbf{d}} & =\dot{\rho} e^{i\left(\psi+\mu_{0}\right)}+i \rho e^{i\left(\psi+\mu_{0}\right)} \dot{\psi}=e^{i \theta} \\
\dot{\mathbf{d}}^{\prime} & =\dot{\rho}^{\prime} e^{i\left(\psi^{\prime}+\mu_{0}\right)}+i \rho^{\prime} e^{i\left(\psi^{\prime}+\mu_{0}\right)} \dot{\psi}^{\prime}=e^{i \theta} .
\end{aligned}
$$

Identifying the real and imaginary terms of (46) and (47) produces the system of equations,

$$
\begin{aligned}
\dot{\rho} & =\cos \left(\theta-\mu_{0}-\psi\right) \\
\dot{\rho}^{\prime} & =\cos \left(\theta-\mu_{0}-\psi^{\prime}\right) \\
\dot{\psi} & =\frac{1}{\rho} \sin \left(\theta-\mu_{0}-\psi\right) \\
\dot{\psi}^{\prime} & =\frac{1}{\rho^{\prime}} \sin \left(\theta-\mu_{0}-\psi^{\prime}\right) \\
\dot{\theta} & =u .
\end{aligned}
$$

In shape coordinates $(\xi, \eta, \alpha, \beta, \phi)$, the system of equations (48)-(52) becomes

$$
\begin{aligned}
\dot{\xi} & =\frac{1}{2}(\sin (\beta+\phi)-\sin (\beta-\phi))=\cos \beta \sin \phi \\
\dot{\eta} & =\frac{1}{2}(\sin (\beta+\phi)+\sin (\beta-\phi))=\sin \beta \cos \phi \\
\dot{\alpha} & =\frac{1}{2}\left(\frac{1}{\xi+\eta} \cos (\beta+\phi)+\frac{1}{\xi-\eta} \cos (\beta-\phi)\right) \\
\dot{\beta} & =\frac{1}{2}\left(\frac{1}{\xi+\eta} \cos (\beta+\phi)-\frac{1}{\xi-\eta} \cos (\beta-\phi)\right) \\
\dot{\phi} & =\dot{\alpha}-u .
\end{aligned}
$$

\section{REFERENCES}

[1] S. T. Besiktepe, P. F. J. Lermusiaux, and A. R. Robinson, "Coupled physical and biogeochemical data-driven simulations of Massachusetts Bay in late summer: Real-time and postcruise sata assimilation," Journal of Marine Systems, vol. 40, pp. 171212, 2003.

[2] P. F. J. Lermusiaux, C. Evangelinos, R. Tian, P. J. Haley, J. J. McCarthy, N. M. Patrikalakis, A. R. Robinson, and H. Schmidt, "Adaptive coupled physical and biogeochemical ocean predictions: A conceptual basis," in Computational Science - ICCS 2004, Part 3, ser. Lecture Notes in Computer Science, vol. 3038, 2004, pp. 685-692.

[3] J. D. Paduan and L. K. Rosenfeld, "Remotely sensed surface currents in Monterey Bay from shore-based HF radar (coastal ocean dynamics application radar)," J. of Geophys. Res. Oceans, vol. 101, no. C9, pp. 20 669-20 686, 1996.

[4] P. F. J. Lermusiaux, "Estimation and study of mesoscale variability in the Strait of Sicily," Dynamics of Atmosphere and Oceans, vol. 29, no. 2-4, pp. 255-303, 1999.

[5] C. H. Pilskaln, C. Lehmann, J. B. Paduan, and M. W. Silver, "Spatial and temporal dynamics in marine aggregate abundance, sinking rate and flux: Monterey Bay, Central California," DeepSea Research Part II-Topical Studies in Oceanography, vol. 45, no. 8-9, pp. 1803-1837, 1998.
[6] W. F. Dabberdt and T. W. Schlatter, "Research opportunities from emerging atmospheric observing and modeling capabilities," Bulletin of the American Meteorological Society, vol. 77, no. 2, pp. 305-323, 1996.

[7] A. C. Lorenc, R. S. Bell, and B. Macpherson, "The meteorological-office analysis correction data assimilation scheme," Quarterly Journal of the Royal Meteorological Society, vol. 117, no. 497, pp. 59-89, 1991.

[8] C. B. Paris, R. K. Cowen, K. M. M. Lwiza, D. P. Wang, and D. B. Olson, "Multivariate objective analysis of the coastal circulation of Barbados, West Indies: Implication for larval transport," Deep-Sea Research Part I - Oceanographic Research Papers, vol. 49, no. 8, pp. 1363-1386, 2002.

[9] J. Willcox, J. Bellingham, Y. Zhang, and A. Baggeroer, "Performance metrics for oceanographic surveys with autonomous underwater vehicles," IEEE Journal Oceanic Engineering, vol. 26, no. 4, pp. 711-725, 2001, special Issue on Autonomous OceanSampling Networks.

[10] A. L. Bertozzi, M. Kemp, and D. Marthaler, "Determining environmental boundaries: Asynchronous communication and physical scales," in Cooperative Control, A Post-Workshop Volume: 2003 Block Island Workshop on Cooperative Control, V. Kumar, N. Leonard, and A. Morse, Eds. Springer, 2005, pp. $35-42$.

[11] D. Marthaler and A. L. Bertozzi, "Tracking environmental level sets with autonomous vehicles," in Recent Developments in Cooperative Control and Optimization, S. Butenko, R. Murphey, and P. Pardalos, Eds. Kluwer Academic Publishers, 2003.

[12] F. Zhang and N. E. Leonard, "Generating contour plots using multiple sensor platforms," in Proceedings of the IEEE Swarm Intelligence Symposium, 2005.

[13] E. Fiorelli, N. E. Leonard, P. Bhatta, D. Paley, R. Bachmayer, and D. Fratantoni, "Multi-AUV control and adaptive sampling in Monterey Bay," in Proceedings IEEE AUV 2004: Workshop on Multiple AUV Operations, 2004.

[14] A. Eliassen, J. S. Sawyer, and S. J., "Upper air network requirements for numerical weather prediction," Technical Note of the World Meteorological Organization, vol. 29, 1954.

[15] L. S. Gandin, Gidrometerologicheskoe Izdatelstvo - Objective Analysis of Meteorological Fields. Leningrad, Jerusalem: English Translation by Israeli Program for Scientific Translations, 1963.

[16] F. P. Bretherton, R. E. Davis, and C. B. Fandry, "A technique for objective analysis and design of oceanographic experiments applied to MODE-73," Deep-Sea Research, vol. 23, pp. 559582, 1976.

[17] C. H. Pilskaln, J. B. Paduan, F. P. Chavez, R. Y. Anderson, and W. M. Berelson, "Carbon export and regeneration in the coastal upwelling system of Monterey Bay, central California," Journal of Marine Research, vol. 54, no. 6, pp. 1149-1178, 1996.

[18] T. Hirawake, T. Odate, and M. Fukuchi, "Long-term variation of surface phytoplankton chlorophylla in the Southern ocean during 1965-2002," Geophysical Research Letters, vol. 32, no. 5 - L05606, 2005.

[19] D. K. Steinberg, M. W. Silver, C. H. Pilskaln, S. L. Coale, and J. B. Paduan, "Midwater zooplankton communities on pelagic detritus (giant larvacean houses) in Monterey Bay, California," Limnology and Oceanography, vol. 39, no. 7, pp. 1606-1620, 1994.

[20] J. Bellingham, "New oceanographic uses of autonomous underwater vehicles," Marine Technology Society Journal, vol. 31, no. 3, pp. 34-47, 1997.

[21] T. B. Curtin, J. G. Bellingham, J. Catipovic, and D. Webb, "Autonomous oceanographic sampling networks," Oceanography, vol. 6, no. 3, pp. 86-94, 1993. 
[22] J. Sherman, R. E. Davis, W. B. Owens, and J. Valdes, "The autonomous underwater glider 'Spray'," IEEE Journal of Oceanic Engineering, vol. 26, no. 4, pp. 437-446, 2001, special Issue on Autonomous Ocean-Sampling Networks.

[23] D. Webb, P. Simonetti, and C. Jones, "SLOCUM: An underwater glider propelled by environmental energy," IEEE Journal Oceanic Engineering, vol. 26, no. 4, pp. 447-452, 2001, special Issue on Autonomous Ocean-Sampling Networks.

[24] C. C. Eriksen, T. J. Osse, T. Light, R. D. Wen, T. W. Lehmann, P. L. Sabin, J. W. Ballard, and A. M. Chiodi, "Seaglider: A long range autonomous underwater vehicle for oceanographic research," IEEE Journal of Oceanic Engineering, vol. 26, no. 4, pp. 424-436, 2001, special Issue on Autonomous OceanSampling Networks.

[25] D. Steere, A. Baptista, D. McNamee, C. Pu, and J. Walpole, "Research challenges in environmental observation and forecasting systems," in Proceedings 6th Int. Cnf. Mobile Computing and Networking (MOBICOMM), 2000, pp. 292-299.

[26] R. Cardell-Oliver, K. Smettem, M. Krantz, and K. Meyer, "Field testing a wireless sensor network for reactive environmental monitoring," in International Conference on Intelligent Sensors, Sensor Networks and Information Processing ISSNIP-04, Melbourne, December 2004.

[27] "Autonomous Ocean Sampling Network, MBARI website, http://www.mbari.org/aosn/," 2003.

[28] D. L. Rudnick, R. E. Davis, C. C. Eriksen, D. M. Fratantoni, and M. J. Perry, "Underwater gliders for ocean research," Marine Technology Society Journal, vol. 38, no. 1, pp. 48-59, 2004.

[29] P. Ögren, E. Fiorelli, and N. E. Leonard, "Cooperative control of mobile sensor networks: Adaptive gradient climbing in a distributed environment," IEEE Transactions Automatic Control, vol. 49, no. 8, pp. 1292-1302, 2004.

[30] E. Fiorelli, P. Bhatta, N. E. Leonard, and I. Shulman, "Adaptive sampling using feedback control of an autonomous underwater glider fleet," in Proceedings of Symposium on Unmanned Untethered Submersible Technology, 2003.

[31] J. Graver, "Underwater gliders: Dynamics, control and design," Ph.D. dissertation, Princeton University, 2005.

[32] P. F. J. Lermusiaux and A. R. Robinson, "Data assimilation via error subspace statistical estimation. Part I: Theory and schemes," Monthly Weather Review, vol. 127, no. 7, pp. 13851407, 1999.

[33] P. E. Lermusiaux, "Data assimilation via error subspace statistical estimation. Part II: Middle Atlantic Bight shelfbreak front simulations and ESSE validation," Monthly Weather Review, vol. 127, no. 7, pp. 1408-1432, 1999.

[34] I. Shulman, C. R. Wu, J. K. Lewis, J. D. Paduan, L. K. Rosenfeld, J. C. Kindle, S. R. Ramp, and C. A. Collins, "High resolution modeling and data assimilation in the Monterey Bay area," Continental Shelf Research, vol. 22, no. 8, pp. 11291151, 2002.

[35] P. F. J. Lermusiaux, "Uncertainty estimation and prediction for the interdisciplinary ocean," J. Comp. Phys., submitted, 2005.

[36] I. Shulman, D. J. McGillicuddy, M. A. Moline, S. H. D. Haddock, J. Kindle, D. Nechaev, and M. W. Phelps, "Biolumineseence intensity modeling and sampling strategy optimization," Journal of Atmospheric and Oceanic Technology, vol. 22, pp. 1267-1281, 2005.

[37] S. R. Ramp, J. D. Paduan, I. Shulman, J. Kindle, F. L. Bahr, and F. Chavez, "Observations of upwelling and relaxation events in the Northern Monterey Bay during august 2000," Journal of Geophysical Research-Oceans, vol. 110, p. Art. No. C07013, 2005.

[38] T. Inanc, S. Shadden, and J. Marsden, "Optimal trajectory generation in ocean flows," in Proceedings 43rd IEEE Conf. Decision and Control, 2004.
[39] S. Berman, "Development of thermocline tracking capabilities for the Slocum underwater glider," Princeton University, Tech. Rep., 2004.

[40] P. F. J. Lermusiaux, D. G. M. Anderson, and C. J. Lozano, "On the mapping of multivariate geophysical fields: Error and variability subspace estimates," Quarterly Journal of the Royal Meteorological Society, vol. 126, no. 565, pp. 1387-1429, 2000.

[41] P. F. J. Lermusiaux, "On the mapping of multivariate geophysical fields: Sensitivities to size, scales, and dynamics," Journal of Atmopsheric and Oceanic Technology, vol. 19, no. 10, pp. 1602-1637, 2002.

[42] A. Bennett, Inverse Modeling of the Ocean and Atmosphere. Cambridge: Cambridge University Press, 2002.

[43] E. Kalnay, Atmospheric Modeling, Data Assimilation and Predictability. Cambridge: Cambridge University Press, 2003.

[44] B. Grocholsky, "Information-theoretic control of multiple sensor platforms," Ph.D. dissertation, The University of Sydney, 2002, available from http://www.acfr.usyd.edu.au.

[45] A. S. Bower, D. M. Fratantoni, W. E. Johns, and H. Peters, "Gulf of Aden eddies and their impact on Red Sea Water," Geophysical Research Letters, vol. 29, pp. 21-1, Nov. 2002.

[46] R. Sepulchre, D. Paley, and N. E. Leonard, "Collective motion in the plane, part I: All-to-all communication," 2005, submitted.

[47] D. Paley, N. E. Leonard, and R. Sepulchre, "Oscillator models and collective motion: Splay state stabilization of self-propelled particles," in Proceedings IEEE Conf. Decision and Control, 2005, submitted.

[48] D. Paley, N. Leonard, and R. Sepulchre, "Collective motion: Bistability and trajectory tracking," in Proceedings 43rd IEEE Conf. Decision and Control, 2004, pp. 1932-1937.

[49] D. Hilbert and S. Cohn-Vossen, Geometry and the Imagination, 2nd ed. Chelsea Pub. Co., 1999.

[50] E. Buckingham, "On physically similar systems; illustrations of the use of dimensional equations," Phys. Rev., vol. 4, pp. 345-376, 1914

[51] H. J. Thiebaux, "Experiment with correlation representations for objective analysis," Monthly Weather Review, vol. 103, pp. 617-627, 1975.

[52] - "Anisotropic correlation functions for objective analysis," Monthly Weather Review, vol. 104, pp. 994-1002, 1976.

[53] W. Curtis, J. Logan, and W. Parker, "Dimensional analysis and the П theorem," Lin. Alg. Appl., vol. 47, pp. 117-126, 1982.

[54] S. Berman, "Estimation of Ocean Field Decorrelation Scales for the Design of Underwater Glider Sampling Trajectories," Undergraduate Senior Thesis, Princeton University, Princeton, 2005.

[55] K. Ide, L. Kuznetsov, and C. K. R. T. Jones, "Lagrangian data assimilation for point vortex systems," Journal of Turbulence, vol. 3, p. Art. No. 053, 2002.

[56] A. Molcard, A. Griffa, and T. M. Ozgokmen, "Lagrangian data assimilation in multilayer primitive equation ocean models," Journal of Atmospheric and Oceanic Technology, vol. 22, no. 1, pp. 70-83, 2005.

[57] A. Molcard, L. I. Piterbarg, A. Griffa, T. M. Ozgokmen, and A. J. Mariano, "Assimilation of drifter observations for the reconstruction of the Eulerian circulation field," J. of Geophys. Research-Oceans, vol. 108, no. C3, p. Art. No. 3056, 2003. 